\title{
DIRECT DECOMPOSITIONS INTO INFINITELY MANY SUMMANDS
}

\author{
BY \\ REINHOLD BAER
}

The theory of direct decompositions of operator loops (or groups) aims at proving criteria for the isomorphy of any two direct decompositions into indecomposable direct summands or more generally for the existence of isomorphic refinements of any two given direct decompositions. We have shown elsewhere $\left.{ }^{1}\right)$ that the central point of the theory is the proof of a fairly sharp refinement theorem which concerns itself with pairs of direct decompositions into two summands each, provided, however, that one restricts attention to decompositions with a finite number of components. It is our object in the present investigation to remove this latter restriction. Since we want to show that the above mentioned special refinement theorem retains its central position even if we concern ourselves with decompositions into infinitely many components, it is only natural to make this special refinement theorem the hypothesis to which we subject the operator loops under consideration. Then we are able to prove two results: (a) the isomorphy of any two direct decompositions into indecomposable direct summands; (b) the existence of isomorphic refinements, provided we assume the validity of the ascending or the descending chain condition in a certain subgroup of the operator center. When proving (a) we work solely with arguments depending on the above mentioned special refinement theorem whereas when proving (b) we have to superimpose upon them arguments of the type used when proving the existence of common refinements of direct decompositions of center free structures.

Isomorphy of direct decompositions may be defined in a variety of ways $\left({ }^{2}\right)$. All definitions used assure center isomorphy. The one we use is slightly stricter; it is a weak form of what we termed elsewhere (Baer $[1$, p. 80]) "exchange isomorphy."

Since the hypotheses as well as contentions of our results are stated in such a way that only terms are used which involve nothing but the partially ordered set of the admissible and normal subloops of the given operator loop, it is natural that we conduct our whole argument within the framework of partially ordered sets $\left({ }^{3}\right)$. We have found that for our present purposes it is ap-

Presented to the Society, April 17, 1948; received by the editors October 1, 1947.

(1) Baer [1] where a discussion of the present state of the theory may be found and which paper should be consulted for further references. Numbers in brackets refer to the bibliography at the end of the paper.

(2) A discussion of the various possibilities for defining isomorphy of direct decompositions may be found in Baer [1].

(3) Such a point of view has been adopted in particular by Ore [1]. 
propriate to consider a complete modular lattice with the following additional property: If $a<b \leqq \sum_{x} x$, then there exists a finite subset $x(1), \cdots, x(n)$ of the set of the $x$ 's such that $a \sum_{i=1}^{n} x(i)<b \sum_{i=1}^{n} x(i)$.

Of previous investigations concerning direct decompositions with an infinity of components we have to mention mainly a result due to Kiokemeister [1] of which our theorem (a) is a generalization and results due to Golowin [1], Kurosh $[1 ; 2]$ of which our theorem (b) constitutes a generalization. $\left(^{4}\right)$

1. The fundamental concepts. In the present section we collect those properties of partially ordered sets which will be needed for our discussion of direct decompositions. The reader should keep in mind that the principal example is the partially ordered set of all the normal and admissible subloops of an operator loop (or subgroups of an operator group).

A. The postulates. We are going to investigate a partially ordered set $P$. The partial ordering we shall indicate by " $<$ " or by " $\leqq$ ". Thus at most one of the three relations: $a<b, a=b, b<a$ is satisfied by the elements $a$ and $b$ in $P$; and $a<b, b<c$ together imply $a<c$.

The partially ordered set $P$ will be subject to a number of requirements of which only one (IV) appears to be new.

I. If $a$ and $b$ are elements in $P$, then there exist (uniquely determined) elements $a b$ and $a+b$ such that $a b \leqq a \leqq a+b, a b \leqq b \leqq a+b$ and $s \leqq a \leqq t$ and $s \leqq b \leqq t$ together imply $s \leqq a b$ and $a+b \leqq t$.

II. If $a \leqq b$, then $a+b c=b(a+c)$ for every $c$ in $P$ (Dedekind's law).

III. If $S$ is a nonvacuous subset of $P$, then there exists one (and only one) element $p$ in $P$ such that $s \leqq p$ for every $s$ in $S$ and $s \leqq q$ for every $s$ in $S$ implies $p \leqq q$.

The element $p$ whose existence has been postulated by III will be called the sum of $S$ and will be denoted, according to the notation used in $S$, by such symbols as: $\sum_{s}$ in $s S$ or $\sum_{\nu} s_{\nu}$ and so on. The existence of finite sums may be deduced, without the help of III, from I; and these will often be denoted by $s_{1}+\cdots+s_{k}$, and so on. We note furthermore that the existence of infinite products can be deduced from I to III, but no explicit use will be made of this fact.

Partially ordered sets meeting requirements I to III have been investigated under various names (modular lattice, structure, if only the validity of I and II is postulated; complete modular lattice, and so on, if I to III are valid); and their elementary properties will be taken for granted. (For these properties and references see, for instance, Birkhoff [1].)

Whereas the preceding properties are self-dual in the sense that with any valid property also its dual holds true, this duality ceases to be true when introducing the next postulate.

$\left.{ }^{4}\right)$ Added October 10, 1948. In the meantime the following important contribution has been published: M. Grayew, Isomorphisms of direct decompositions in Dedekind structures, Bull. Acad. Sci. URSS. Ser. Math. vol. 11 (1947) pp. 33-46. In spite of a natural overlap his hypotheses, methods and results appear to be essentially different from ours. 
IV. If $u<v \leqq \sum_{8}$ in $s s$, then there exists a finite number of elements $s_{1}, \cdots, s_{k}$ in $S$ such that $u\left(s_{1}+\cdots+s_{k}\right)<v\left(s_{1}+\cdots+s_{k}\right)$.

We add one further postulate which serves only the convenience of notation without involving any real loss in generality.

$\mathrm{V}$. There exists an element $o$ in $P$ such that $o \leqq x$ for every $x$ in $P$.

If $\mathrm{V}$ were not true, then we would investigate instead of the whole system $P$ only the set of all the elements $x$ in $P$ containing a suitably selected element $v$ in which case $v$ would take the place of $o$.

The most important example of a partially ordered set meeting requirements $\mathrm{I}$ to $\mathrm{V}$ is furnished by the system of all the admissible and normal subloops of an operator loop $\left(^{5}\right)$. Another example is provided by the system of all the open subsets of a topological space; in this case IV is satisfied in an even stricter form $(k=1)$. As a further example we mention the open and normal subgroups of a topological group. The closed and normal subgroups of a topological group will, in general, not satisfy postulate IV.

Throughout we shall investigate a definitely given partially ordered set $P$ which meets requirements I to $\mathrm{V}$. If $u$ and $v$ are elements in $P$ such that $u \leqq v$, then we denote by $v / u$, as is customary, the system of all the elements $x$ in $P$ such that $u \leqq x \leqq v$. It is readily seen that $v / u$ is likewise a partially ordered set meeting requirements $\mathrm{I}$ to $\mathrm{V}$.

Isomorphism. If $M$ and $N$ are partially ordered sets, then we term isomorphism of $M$ upon $N$ any one-to-one and monotone increasing mapping of the whole system $M$ upon the whole system $N$. For instance, it is well known that an isomorphism of $(a+b) / a$ upon $b / a b$ may be obtained by mapping the element $x$ between $a$ and $a+b$ upon the element $b x$ between $a b$ and $b$.

Notation. Instead of " $u / o$ " we shall say shortly " $u$." Thus $u$ may stand both for a definite element in $P$ and for the set of all the elements $x$ in $P$ such that $x \leqq u$. There will not be any danger of confusion.

B. Direct sums.

Definition 1. If $a, b, c$ are elements in $P$ such that $a=b+c$ and $o=b c$, then $a$ is the direct sum $b \oplus c$ of $b$ and $c$.

This is the customary definition.

LEMma 1. If $a \leqq b \leqq a \oplus c$, then $b=a \oplus b c$.

Proof. $a(b c)=(a c) b=o b=o$ and $b=b(a+c)=a+b c$ by II.

Corollary 1. If $a \oplus b=a \oplus c$, and if $x^{\prime}=c(a+x)$ for $x \leqq b$, then $a \oplus x$ $=a \oplus x^{\prime}$, and mapping $x$ upon $x^{\prime}$ constitutes an isomorphism of $b$ upon $c$.

Proof. $a x \leqq a b=o, a x^{\prime}=a c(a+x)=o(a+x)=o, a+x^{\prime}=a+c(a+x)=(a+c)$ $\cdot(a+x)=(a+b)(a+x)=a+x$ by II. Hence $a \oplus x=a \oplus x^{\prime}$. Furthermore $b\left(a+x^{\prime}\right)$ $=b(a+x)=a b+x=x$ and now it is clear how to complete the proof.

(5) See, for instance, Baer [1]. 
REMARK. These facts are, of course, well known, and in particular the isomorphy mentioned in Corollary 1 is nothing but a special case of the well known isomorphy of $(a+b) / a$ and $b / a b$.

DEFINITION 2. If the subset $S$ of $P$ contains at least two elements, if $s^{\prime}$ is, for every element $s$ in $S$, the sum of all the elements, not $s$, in $S$, and if $s s^{\prime}=0$ for every $s$ in $S$, then the sum of the elements in $S$ is their direct sum.

In a more formal way we may restate Definition 2, using Definition 1, as follows:

$a$ is the direct sum of $S$, if $a=s \oplus s^{\prime}$ for every $s$ in $S$.

Using the convention that the sum of the vacuous set is $o$, Definition 2 may be extended so as to apply to any subset $S$ of $P$.

The direct sum of $S$ will be indicated in a variety of ways, depending on the way $S$ has been given, namely $\sum_{s \in S}^{*} s$, or $\sum_{\nu}^{*} s_{v}$, or $s(1) \oplus \cdots \oplus s(i)$, and so on.

REMARK. Suppose that there are given an element $a$ and a set $H$ of pairs $\left(s, s^{\prime}\right)$ of elements $s$ and $s^{\prime}$ in $P$, meeting the following requirements:

(a) $a=s \oplus s^{\prime}$ for every $\left(s, s^{\prime}\right)$ in $H$;

(b) If $\left(s, s^{\prime}\right)$ and $\left(t, t^{\prime}\right)$ are different pairs in $H$, then $t \leqq s^{\prime}$ (and $s \leqq t^{\prime}$ ).

One verifies readily that the pairs $\left(s, s^{\prime}\right)$ appearing in our Definition 2 satisfy conditions (a) and (b); and one may prove conversely that $a$ is the direct sum of the first members of the pairs in $H$, provided $H$ is finite. But for infinite $H$ there exist some types of "generalized direct sums" which are different from our Definition 2. Parts of the theory which we are going to develop hold also for these generalized direct sums.

Corollary 2. If $a \oplus b=a \oplus \sum_{s \in S}^{*} s$, and if $s^{*}=b(a+s)$ for $s$ in $S$, then $a \oplus s=a \oplus s^{*}$ for $s$ in $S$ and $b=\sum_{s \in S}^{*} s^{*}$.

This is a fairly immediate consequence of Corollary 1 , if one notes that isomorphisms map direct sums upon direct sums.

Lemma 2. Suppose that $S$ is a subset of $P$, and that there is given a division of $S$ into mutually exclusive and exhaustive subsets $S(\nu)$. Denote by $a$ and $a(\nu)$ the sum of $S$ and $S(\nu)$ respectively. Then $a$ is the direct sum of $S$ if, and only if, every $a(\nu)$ is the direct sum of $S(\nu)$ and $a=\sum_{\nu}^{*} a(\nu)$.

Proof. Denote by $T(\nu)$ the set of all the elements in $S$, but not in $S(\nu)$; and denote by $b(\nu)$ the sum of the elements in $T(\nu)$. Then

$$
a=a(\nu)+b(\nu) \text { and } b(\nu)=\sum_{\mu \neq \nu} a(\mu) .
$$

A. Assume that $a$ is the direct sum of $S$. Then it is immediately clear that $a(\nu)$ is the direct sum of $S(\nu)$.

Suppose next that the elements $s(1), \cdots, s(m)$ belong to $S(\nu)$ and that the elements $t(1), \cdots, t(n)$ belong to $T(\nu)$. Then we prove by complete 
induction with respect to $n$ that

$$
(s(1)+\cdots+s(m))(t(1)+\cdots+t(n))=o .
$$

This is certainly true for $n=0$; and thus we may assume the validity of $(n-1) \quad(s(1)+\cdots+s(m))(t(1)+\cdots+t(n-1))=o$.

Since $a$ is the direct sum of $S$, and since the $s(i)$ and $t(j)$ are distinct elements in $S$, it follows furthermore that

$$
(s(1)+\cdots+s(m)+t(1)+\cdots+t(n-1)) t(n)=o .
$$

Consequently we may infer from II that $\left({ }^{6}\right)$

$$
\begin{aligned}
(s(1)+\cdots+ & s(m))(t(1)+\cdots+t(n)) \\
= & (s(1)+\cdots+s(m))(s(1)+\cdots+s(m)+t(1)+\cdots \\
& +t(n-1))(t(1)+\cdots+t(n)) \\
= & (s(1)+\cdots+s(m))[t(1)+\cdots+t(n-1)+t(n)(s(1)+\cdots \\
& +s(m)+t(1)+\cdots+t(n-1))] \\
= & (s(1)+\cdots+s(m))(t(1)+\cdots+t(n-1))=o,
\end{aligned}
$$

completing the inductive proof of $(n)$.

If $a(\nu) b(\nu) \neq 0$, then we would infer from (IV) and $o<a(\nu) \sum_{t} \in T_{T(\nu)} t$ the existence of a finite number of elements $t(1), \cdots, t(n)$ in $T(\nu)$ such that $o<a(\nu)(t(1)+\cdots+t(n))$; and we would now infer from (IV) and $o<(t(1)$ $+\cdots+t(n)) \sum_{s} \in_{S(\nu)} s$ the existence of a finite number of elements, $s(1), \cdots, s(m)$, such that $o<(t(1)+\cdots+t(n))(s(1)+\cdots+s(m))$. But this contradicts $(n)$. Hence $a(\nu) b(\nu)=o$; and we have shown that $a=\sum_{\nu}^{*} a(\nu)$, completing the proof of the necessity of our conditions.

B. Assume conversely the validity of our conditions. Then we have certainly $a=a(\nu) \oplus b(\nu)$ for every $\nu$. If $s$ is an element in $S(\nu)$, and if $r$ is the sum of all the elements, not $s$, in $S(\nu)$, then $a(\nu)=s \oplus r$, since $a(\nu)$ is the direct sum of the elements in $S(\nu)$. Hence it follows from an argument used in the first part of the proof that $a=a(\nu) \oplus b(\nu)=(s \oplus r) \oplus b(\nu)=s \oplus(r \oplus b(\nu))=s \oplus s^{\prime}$; and thus we have shown that $a$ is the direct sum of $S$, completing the proof.

CoRollary 3. If $a \oplus b=a \oplus \sum_{s \in S}^{*} s$, if $U$ is a subset of $S$ and $V$ the set of elements in $S$ which do not belong to $U$, then

$$
a \oplus b=a \oplus \sum_{u \in U}^{*} u \oplus \sum_{v \in V}^{*} b(a+v) .
$$

This is easily deduced from Corollary 2 and Lemma 3.

C. The center of an element. Kurosh [1, p. 190] has introduced the "center of a pair of direct decompositions." For our purposes it will be more con-

(6) This is a well known argument. 
venient to form, roughly speaking, the sum of all these "centers."

Definition 3. The center $z(x)$ of the element $x$ in $P$ is the sum of all the elements $e(d+a)(d+b)$ such that $x=d \oplus e=a \oplus b$.

The existence of the element $z(x)$ is assured by Postulate III. It is clear that $z(x) \leqq x$.

REMARK $\left.{ }^{7}\right)$. Suppose that $P$ is the set of all the normal and admissible subloops of the operator loop $L$, and that $L=A \oplus B=D \oplus E$. Denote by $\alpha, \beta$ and $\delta, \epsilon$ the pairs of complementary decomposition endomorphisms of $L$ such that $A=L \alpha, B=L \beta, D=L \delta$ and $E=L \epsilon$. Then $L \delta \alpha \epsilon$ is known to be (Baer [1, p. 67]) part of the operator center of $L$. But

$$
\begin{aligned}
L \delta \alpha \epsilon & =D \alpha \epsilon=[(B+D) \cap A] \epsilon=[D+((B+D) \cap A)] \cap E \\
& =(B+D) \cap(D+A) \cap E ;
\end{aligned}
$$

and thus we have seen that the "center" in the sense of Definition 3 is part of the operator center of $L$.

It should be noted furthermore that the center in the sense of Definition 3 may be smaller than the operator center. If $L$ is, for instance, a cyclic group of order a prime, then $L$ is its own operator center whereas its center according to Definition 3 is $\boldsymbol{o}$. For this reason we should have termed $z(x)$ the "decomposition center of $x$." But since there does not arise any danger of confusion, we have preferred the shorter expression.

LEMmA 3. If $a \oplus b=a \oplus c=d$, then $b \leqq c+z(d)$.

Proof. It follows from $c \leqq b+c \leqq d=c \oplus a$ and Lemma 1 that $b+c=c$ $\oplus a(b+c)$. But $a(b+c)=a(b+c)(a+c) \leqq z(d)$; and thus $b \leqq b+c=c+a(b+c)$ $\leqq c+z(d)$, as we claimed.

REMARK. In the theory of operator loops one shows (Baer [1, p. 69]) that $D=A \oplus B=A \oplus C$ implies the "center-isomorphy" of the subloops $B$ and $C$ of $D$. Lemma 3 and Corollary 1 together express a kind of substitute for this important fact.

Lemma 4. If $a \oplus b=c$, then $z(a) \leqq z(c)$; and $z(a)=z(c)$ implies $z(b)=0$.

Proof. If $a=u \oplus v=e \oplus \dot{f}$, then it follows from Lemma 2 that $c=u \oplus(v \oplus b)$ $=e \oplus(f \oplus b)$. Hence

$$
u(v+e)(v+f) \leqq u[(v+b)+e][(v+b)+(b+f)] \leqq z(c),
$$

proving that $z(a) \leqq z(c)$.

If $z(a)=z(c)$, then it follows from what we showed already that

$$
z(b)=b z(b) \leqq b z(c)=b z(a) \leqq b a=o \quad \text { or } \quad z(b)=o .
$$

REMARK. It follows from Lemma 4 that $z(a) \leqq a z(c)$, if $a$ is a direct sum-

(7) In this remark, as in similar discussions below, we shall use notations and results of Baer [1] without detailed restatement. 
mand of $c$. If $A$ is a direct summand of the operator loop $L$, then the operator center of $A$ is exactly the intersection of $A$ and the operator center of $L$. We note furthermore the possibility that both $z(a)$ and $z(b)$ are $o$, though $z(a \oplus b) \neq o$; as an example let $a$ and $b$ be cyclic groups of order $p$ in which case $z(a)=z(b)=o$, though $a \oplus b=z(a \oplus b)$.

D. Strong refinability.

Definition 4. The element $p$ is S-refinable, if $p=a \oplus b=d \oplus e$ implies $p=a d \oplus a e \oplus b d \oplus b e$.

Theorem 1. The element $p$ is S-refinable if, and only if, $z(p)=o$.

Proof. Suppose first that $p$ is $S$-refinable and that $p=a \oplus b=d \oplus e$. Then $a=a p=a(a d \oplus a e \oplus b d \oplus b e)=a d+a e+a(b d+b e)=a d+a e$ by Dedekind's law and the fact that $a(b d+b e) \leqq a b=o$. Likewise $d=a d+b d$ and $b=b d+b e$. Consequently

$$
\begin{aligned}
a(b+d)(b+e) & =a(b+a d+b d)(b+e) \\
& =a(b+a d)(b+e)=(a d+a b)(b+e) \\
& =a d(b+e)=a d(b e+b d+e) \\
& =a d(e+b d)=a(d e+b d)=a b d=o
\end{aligned}
$$

proving that $z(p)=o$.

Assume conversely that $z(p)=o$, and that $p=a \oplus b=d \oplus e$. We note first that, by Dedekind's law, $a d(a e+b d+b e)=a[b d+d(a e+b e)]=a b d=o$; and now it follows from this (and three similar equations) that the sum of $a d, a e, b d$ and $b e$ is certainly their direct sum. Next we infer from $z(p)=0$ that

$$
\begin{aligned}
a & =a+b(a+d)(a+e)=[a+b(a+d)](a+e) \\
& =(a+b)(a+d)(a+e)=(a+d)(a+e) \\
& =a+d(a+e) ;
\end{aligned}
$$

and this implies $d(a+e) \leqq a$ or $d a \leqq d(a+e) \leqq d a$ or $d a=d(a+e)$. We show likewise that $a e=e(a+d)$ and deduce from Dedekind's law and $p=d+e$ that

$$
\begin{aligned}
a \leqq(a+e)(a+d) & =(d+e)(a+e)(a+d) \\
& =[d(a+e)+e](a+d) \\
& =d(a+e)+e(a+d)=d a+a e .
\end{aligned}
$$

Since we may show likewise that $b \leqq d b+b e$, it follows that

$$
p=a+b \leqq d a+a e+d b+b e \leqq p ;
$$

and this completes the proof of the fact that $p=d a \oplus a e \oplus d b \oplus b e$. Thus $z(p)=o$ implies $S$-refinability of $p$. 
CoRollary 4. S-refinability of $p$ implies $S$-refinability of every direct sum. mand of $p$.

Proof. If $p$ is $S$-refinable, then $z(p)=o$ by Theorem 1. If $d$ is a direct summand of $p$, then $z(d) \leqq z(p)$ by Lemma 4 . Hence $z(d)=0$ and $S$-refinability of $d$ is a consequence of Theorem 1 .

THEorem 2. If $p=\sum_{s \in S}^{*} s=\sum_{t \in T}^{*} t$ is S-refinable, then $p=\sum_{s, t}^{*}$ st.

This theorem asserts that any two direct decompositions of an $S$-refinable element possess a common refinement. For future applications it will be convenient to prove first a more general proposition.

Lemma 5. If $p=a \oplus b=\sum_{s \in S}^{*} s$, and if $a z(p)=o$, then $b=\sum_{s \in S}^{*}(b s)$.

Proof. We shall proceed in a number of steps.

A. $p=d \oplus e$. Then $a(b+d)(b+e) \leqq a z(p)=o$ or $a(b+d)(b+e)=o$. Hence $(b+d)(b+e)=b \oplus a(b+d)(b+e)=b$ by Lemma 1 . Consequently

$$
\begin{aligned}
b d \oplus b e & =d(b+d)(b+e)+e(b+d)(b+e) \\
& =d(b+e)+e(b+d) \\
& =[d(b+e)+e](b+d) \quad \text { (by Dedekind's law) } \\
& =(d+e)(b+e)(b+d)=(b+e)(b+d) \\
& =b,
\end{aligned}
$$

completing the proof of our lemma, in case $S$ consists of two elements.

B. $p=s(1) \oplus \cdots \oplus s(n)$ for $n$ a positive integer. Then we may proceed by complete induction with respect to $n$, since our contention is trivially valid for $n=1$. Hence we may assume validity of our proposition for decompositions of $p$ into $n-1$ direct summands. It follows from Lemma 2 that

$$
p=s(1) \oplus \cdots \oplus s(n-2) \oplus s(0) \text { for } s(0)=s(n-1) \oplus s(n)
$$

is a direct decomposition of $p$ into a direct sum of $n-1$ summands; and thus it follows from our induction hypothesis that

$$
b=\sum_{i=0}^{n-2} *(s(i) b) .
$$

Let $q=s(1) \oplus \cdots \oplus s(n-1)$. Then $p=q \oplus s(n)$, and it follows from what we showed under A that $b=b q \oplus b s(n)$. Consequently

$$
\begin{aligned}
b s(0) & =(b q \oplus b s(n))(s(n-1) \oplus s(n)) \\
& =b \sum_{i=1}^{n-1} s(i)[s(n-1) \oplus s(n)]+b s(n) \quad \text { (by Dedekind's law) } \\
& =b s(n-1) \oplus b s(n),
\end{aligned}
$$


since $p=s(1) \oplus \cdots \oplus s(n-1) \oplus s(n)$, and hence

$$
b=\sum_{i=1}^{n} *(s(i) b),
$$

completing the proof of our lemma in case $S$ is a finite set.

C. $p=\sum_{s \in S}^{*} s$. We form the sum $s^{*}$ of the elements $b s$ for $s$ in $S$. It is obvious that $s^{*}$ is the direct sum of the elements $b s$ for $s$ in $S$ and that $s^{*} \leqq b$. If $s^{*}$ and $b$ were different, then we would have

$$
s^{*}<b \leqq p=\sum_{s \in S}^{*} s .
$$

Thus there exists by Postulate IV a finite number of elements $s(1), \cdots$, $s(n-1)$ in $S$ such that

$$
\sum_{i=1}^{n-1} *(s(i) b)=s^{*} \sum_{i=1}^{n-1} * s(i)<b \sum_{i=1}^{n-1} * s(i) .
$$

Denote by $s(n)$ the sum of all those elements in $S$ which are different from $s(1), \cdots, s(n-1)$. Then $p=s(1) \oplus \cdots \oplus s(n-1) \oplus s(n)$ by Lemma 2 . We infer from what we showed under $\mathrm{B}$ that $b=\sum_{i=1}^{* n}(s(i) b)$. Hence

$$
\sum_{i=1}^{n-1} *(s(i) b)<b \sum_{i=1}^{n-1} * s(i)=\sum_{i=1}^{n} *(s(i) b) \sum_{i=1}^{n-1} * s(i)=\sum_{i=1}^{n-1} *(s(i) b)
$$

by Dedekind's law and Lemma 2 . Thus our hypothesis $s^{*} \neq b$ has led us to a contradiction which completes the proof.

Proof of Theorem 2. Since $p$ is $S$-refinable, we infer $z(p)=o$ from Theorem 1. If $p=a \oplus b=\sum_{s \in S}^{*} s$, then we have certainly $a z(p)=o$, and thus it follows from Lemma 5 that $b=\sum_{s \in S}^{*}(b s)$. Since this result applies on every direct summand $b$ of $p$, we infer from $p=\sum_{s \in S}^{*} s=\sum_{t \in T^{t}}^{*}$ that $t=\sum_{s \in S}^{*}(s t)$ for every $t$ in $T$, and we infer from Lemma 2 that $p=\sum_{s, t}^{*}(s t)$ as we claimed.

E. Weak refinability. The type of strong refinability introduced by Definition 4 is of comparatively rare occurrence. Thus we introduce now a weak type of refinability which is realized in a great number of important instances.

Definition 5. The element $p$ in $P$ is (weakly) refinable if every direct summand $d$ of $p$ has the following property:

If $d=a \oplus b=e \oplus f$, then there exist direct decompositions

$$
a=a^{\prime} \oplus a^{\prime \prime}, \quad b=b^{\prime} \oplus b^{\prime \prime}, \quad e=e^{\prime} \oplus e^{\prime \prime}, \quad f=f^{\prime} \oplus f^{\prime \prime}
$$

such that

$$
\begin{aligned}
& a^{\prime} \oplus b^{\prime}=b^{\prime} \oplus e^{\prime}=e^{\prime} \oplus f^{\prime}=f^{\prime} \oplus a^{\prime}=d^{\prime}, \\
& a^{\prime \prime} \oplus b^{\prime \prime}=b^{\prime \prime} \oplus f^{\prime \prime}=f^{\prime \prime} \oplus e^{\prime \prime}=e^{\prime \prime} \oplus a^{\prime \prime}=d^{\prime \prime} .
\end{aligned}
$$

We note first a few simple consequences of (5.2) and Lemma 2: 
$d=d^{\prime} \oplus d^{\prime \prime}=\left(d^{\prime \prime} \oplus b^{\prime}\right) \oplus a^{\prime}=\left(d^{\prime \prime} \oplus b^{\prime}\right) \oplus e^{\prime}$. It follows from Lemma 3 that $a^{\prime} \leqq e^{\prime}+z(d)$; and thus $a^{\prime}$ and $e^{\prime}$ are "center isomorphic."

It may be deduced from Corollary 4 that $S$-refinability implies the weak refinability in the sense of Definition 5. Usually we shall say "refinable" instead of "weakly refinable"; and there will not arise any danger of confusion, since we shall always emphasize it whenever we speak of $S$-refinability.

It is trivial that refinability of $p$ implies the refinability of every direct summand of $p$, and that isomorphy of $p$ and $q$ and refinability of $p$ imply the refinability of $q$.

Elsewhere (Baer [1, p. 77]) we have exhibited a large class of operator loops which are refinable in the sense of Definition 5 .

THEOREM 3. If $p$ is refinable, and if

$$
p=a(1) \oplus \cdots \oplus a(m)=b(1) \oplus \cdots \oplus b(n)
$$

are direct decompositions of $p$ into a finite number of direct summands, then there exist direct decompositions

$$
\begin{aligned}
& a(i)=a(i, 1) \oplus \cdots \oplus a(i, n), \\
& b(j)=b(j, 1) \oplus \cdots \oplus b(j, m)
\end{aligned}
$$

with the following properties:

(5.E) If $1 \leqq k \leqq m$, and if $J$ is a set of integers between 1 and $n$, then

$$
p=\sum_{i \neq k}^{*} a(i) \oplus \sum_{j \in J}^{*} a(k, j) \oplus \sum_{j} \notin_{J}^{*} b(j, k) .
$$

We omit the proof, since the arguments which we used elsewhere (Baer [1], when reducing the General Refinement Theorem from the Special Refinement Theorem, pp. 81-84) may be carried over almost verbally to the present situation (as has been pointed out op. cit.).

2. Decomposition into indecomposable direct summands. It is the object of the present section to obtain a generalization of $\S 1$, Theorem 3 so as to apply to decompositions into infinitely many direct summands. This greater generality we can attain only by restricting our attention to decompositions into indecomposable direct summands.

A. Exchange of one indecomposable direct summand. We begin with the following obvious definition.

DeFINITION 1. The element $u$ is indecomposable, if $o$ and $u$ are its only direct summands, and if $o<u$.

Note that $z(u)=o$ is a consequence of the indecomposability of $u$.

LemMA 1. If $p$ is refinable, if $p=a \oplus b=\sum_{s \in S}^{*} s$, and if $a$ and the elements $s$ in $S$ are indecomposable, then there exists an element $u$ in $S$ such that $p=a \oplus u^{\prime}$ (where $u^{\prime}=\sum_{s \neq u}^{*} s$ is the sum of the elements $s$, not $u$, in $S$ ).

Proof. From $o<a \leqq \sum_{s \in S}$ and Postulate IV we infer the existence of 
a finite number of elements $s(1), \cdots, s(n)$ in $S$ such that

$$
o<a \sum_{i=1}^{n} * s(i) \text {. }
$$

Denote by $s(0)$ the sum of all the elements $s$ in $S$ which are different from every $s(i)$. Then we infer from $\S 1$, Lemma 2 that

$$
p=a \oplus b=s(0) \oplus s(1) \oplus \cdots \oplus s(n) .
$$

We apply 1, Theorem 3 upon these two direct decompositions (2) of p. Consequently there exist direct decompositions

$$
a=\sum_{i=0}^{n} * a(i), \quad b=\sum_{i=0}^{n} * b(i)
$$

such that

$$
p=\sum_{i \neq j}^{*} s(j) \oplus a(i) \oplus b(i) \quad \text { for } i=0,1, \cdots, n \text {. }
$$

(Note that we did not exhaust in the least the contents of $\S 1$, Theorem 3.)

It follows from (4) that $o=a(0) \sum_{i=1}^{* n} s(i)$; and thus it follows from (1) that $a \neq a(0)$. But $a(0)$ is, by (3), a direct summand of the indecomposable element $a$. Hence

$$
o=a(0) .
$$

Now it follows from (5) and the indecomposability of $a$ that one and only one $a(i)$ with $0<i$ is different from $o$; and thus we may assume without loss in generality that

$$
o=a(0)=a(1)=\cdots=a(n-1), \quad a=a(n) .
$$

It follows from (4) and Corollary 1 that $s(n), p / \sum_{i=0}^{* n-1} s(i)$ and $a(n) \oplus b(n)$ $=a \oplus b(n)$ are isomorphic. Since $s(n)$ is indecomposable, so is $a \oplus b(n)$. Hence $b(n)=o$ is a consequence of $a \neq o$. Now it follows from (4) and (5') that

$$
p=\sum_{i=0}^{n-1} * s(i) \oplus a=s(n)^{\prime} \oplus a
$$

where $s(n)^{\prime}$ is the sum of all the elements $s \neq s(n)$ in $S$. This completes the proof.

Corollary 1. If $p$ is refinable, if $p=a \oplus b \oplus c=c \oplus \sum_{\nu}^{*} d(\nu)$, and if $a$ and the elements $d(\nu)$ are indecomposable, then there exists $a \quad \tau$ such that $p=c \oplus \sum_{\nu \neq \tau}^{*} d(\nu) \oplus a$.

Proof. Let $d^{*}(\nu)=(a+b)(c+d(\nu))$. Then it follows from $\$ 1$, Corollary 2 that $c \oplus d(\nu)=c \oplus d^{*}(\nu)$ for every $\nu$ and $a \oplus b=\sum_{\nu}^{*} d^{*}(\nu)$. It follows from $\S 1$, Corollary 1 that $d(\nu),(d(\nu) \oplus c) / c=\left(d^{*}(\nu) \oplus c\right) / c$ and $d^{*}(\nu)$ are isomorphic; and 
thus the indecomposability of $d(\nu)$ implies the indecomposability of $d^{*}(\nu)$. We infer from Lemma 1 the existence of a $\tau$ such that

$$
a \oplus b=\sum_{\nu \neq \tau} * d^{*}(\nu) \oplus a,
$$

and this implies that

$$
p=a \oplus b \oplus c=a \oplus c \oplus \sum_{\nu \neq \tau}^{*} d^{*}(\nu)=a \oplus c \oplus \sum_{\nu \neq \tau}^{*} d(\nu),
$$

since $c \oplus d(\nu)=c \oplus d^{*}(\nu)$. This completes the proof.

LEMMA 2. If $p$ is refinable and the direct sum of indecomposable direct summands, then every direct summand, not $o$, of $p$ possesses an indecomposable direct summand.

Proof. Assume that $p=a \oplus b=\sum_{s \in S}^{*} s$ where $a \neq 0$ and where every $s$ is indecomposable. From $o<a \leqq p=\sum_{s \in S}^{*} S$ and Postulate IV we infer the existence of a finite number of elements $s(1), \cdots, s(n)$ in $S$ such that

$$
o<a \sum_{i=1}^{n} * s(i)
$$

We let $e=\sum_{i=1}^{* n} s(i)$ and we denote by $f$ the (direct) sum of all the elements $s$ in $S$ which are different from every $s(i)$. Then

$$
p=a \oplus b=e \oplus f
$$

by $\$ 1$, Lemma 2 ; and we infer from the refinability of $p$ (see $\S 1$, Definition 5 ) the existence of decompositions

$$
a=a^{\prime} \oplus a^{\prime \prime}, \quad b=b^{\prime} \oplus b^{\prime \prime}, \quad e=e^{\prime} \oplus e^{\prime \prime}, \quad f=f^{\prime} \oplus f^{\prime \prime}
$$

such that

$$
\begin{aligned}
& a \oplus b^{\prime}=b^{\prime} \oplus e^{\prime}=e^{\prime} \oplus f^{\prime}=f^{\prime} \oplus a^{\prime}=p^{\prime}, \\
& a^{\prime \prime} \oplus b^{\prime \prime}=b^{\prime \prime} \oplus f^{\prime \prime}=f^{\prime \prime} \oplus e^{\prime \prime}=e^{\prime \prime} \oplus a^{\prime \prime}=p^{\prime \prime} .
\end{aligned}
$$

We prove:

$$
o \neq a^{\prime} \text { and } o \neq e^{\prime} \text {. }
$$

If this were not true, then we would infer from the first set of equations (4) that $0=a^{\prime}=e^{\prime}$. This would imply, because of (4), (3), $o=a^{\prime \prime} e^{\prime \prime}=a e$, contradicting (1). Thus (5) is true.

We apply $\$ 1$, Theorem 3 on the direct decompositions

$$
e=e^{\prime} \oplus e^{\prime \prime}=\sum_{i=1}^{n} * s(i)
$$

this is possible, since $e$ is refinable as a direct summand of the refinable ele- 
ment $p$. Consequently there exist direct summands $s^{\prime}(i)$ of $s(i)$, for $i=1, \cdots, n$, such that

$$
e=s^{\prime}(1) \oplus \cdots \oplus s^{\prime}(n) \oplus e^{\prime \prime}
$$

Since the $s(i)$ are indecomposable, we have either $s^{\prime}(i)=o$ or $s^{\prime}(i)=s(i)$. Consequently we may assume without loss in generality that there exists an integer $k, 0 \leqq k \leqq n$, such that $s^{\prime}(i)=s(i)$ for $i \leqq k$ and $s^{\prime}(i)=o$ for $k<i$. If $k=0$, then it would follow from (7) that $e=e^{\prime \prime}$. This would imply $e^{\prime}=0$ which is impossible by (5). Hence $0<k$.

Let $t(i)=e^{\prime}\left(s(i)+e^{\prime \prime}\right)$ for $i=1, \cdots, k$. Then it follows from (6), (7) and $\S 1$, Corollary 2 that $e^{\prime \prime} \oplus s(i)=e^{\prime \prime} \oplus t(i)$ for $i=1, \cdots, k$ and $e^{\prime}=\sum_{i=1}^{* k} t(i)$.

Next we let $a(i)=a^{\prime}\left(t(i)+b^{\prime}\right)$ for $i=1, \cdots, k$. Since $b^{\prime} \oplus a^{\prime}=b^{\prime} \oplus e^{\prime}$ $=b^{\prime} \oplus \sum_{i=1}^{* k} t(i)$, by (4), we infer from $\$ 1$, Corollary 2 , that $b^{\prime} \oplus t(i)=b^{\prime} \oplus a(i)$ and $a^{\prime}=\sum_{i=1}^{* k} a(i)$.

Finally we infer from $\S 1$, Corollary 1 successively the isomorphy of $a(i)$, $\left(a(i) \oplus b^{\prime}\right) / b^{\prime}=\left(t(i) \oplus b^{\prime}\right) / b^{\prime}, \quad t(i), \quad\left(t(i) \oplus e^{\prime \prime}\right) / e^{\prime \prime}=\left(s(i) \oplus e^{\prime \prime}\right) / e^{\prime \prime}, \quad s(i)$. Since $s(i)$ is indecomposable, so is $a(i)$. But $a(i)$ is a direct summand of the direct summand $a^{\prime}$ of $a$. Since $0<k$, we have established the existence of indecomposable direct summands of $a$, as we claimed.

REMARK. The interesting question whether every direct summand, not $o$, of a refinable element which is the direct sum of indecomposable elements is itself the direct sum of indecomposable elements appears still to be undecided.

B. Exchange of a finite number of indecomposable direct summands. In Lemma 1 we established the possibility of exchanging an indecomposable direct summand for some member of a direct decomposition into indecomposable direct summands. In our next propositions we shall establish similar possibilities for direct summands which are direct sums of a finite number of indecomposable direct summands.

LEMMA 3. If $p$ is refinable, if $p=\sum_{s \in S}^{*} s=b \oplus \sum_{i=1}^{* n} a(i)$ where the $a(i)$ and the $s$ in $S$ are indecomposable, then there exist elements $s(1), \cdots, s(n)$ in $S$ such that $p=\sum_{i=1}^{* j} a(i) \oplus t(j)$ for $j=1, \cdots, n$ where $t(j)$ is the sum of the elements $s$ in $S$ which are different from every $s(i)$ with $0<i \leqq j$.

Proof. Since the contention of Lemma 3 is certainly true, if $n=0$, we may make the induction hypothesis that Lemma 3 is valid for $n-1$. Since $p=(a(n)$ $\oplus b) \oplus \sum_{i=1}^{* n-1} a(i)$, we infer from the induction hypothesis the existence of elements $s(1), \cdots, s(n-1)$ in $S$ such that $p=\sum_{i=1}^{* j} a(i) \oplus t(j)$ for $0<j<n$. Let $c=\sum_{i=1}^{* n-1} a(i)$. Then

$$
p=b \oplus c \oplus a(n)=c \oplus t(n-1)
$$

where $t(n-1)$ is a direct sum of indecomposable elements in $S$ and where $a(n)$ is indecomposable. Thus we may apply Corollary 1 . Hence there exists an element $s(n)$ in $S$ which is different from $s(1), \cdots, s(n-1)$ (for $s(n)$ is 
one of the summands of $t(n-1))$ such that

$$
p=c \oplus a(n) \oplus t(n)
$$

where $t(n)$ is the direct sum of all those elements in $S$ which are different from $s(1), \cdots, s(n-1)$ and $s(n)$. Thus $p=\sum_{i=1}^{* n} a(i) \oplus t(n)$, completing the induction.

Corollary 2. Suppose that $p$ is refinable, that $p=\sum_{s \in S}^{*} s$ where the elements $s$ in $S$ are indecomposable, and that, for every positive integer $i$, there exist elements $a(i), b(i)$ such that $a(i)$ is indecomposable and $p=\sum_{i=1}^{* n} a(i) \oplus b(n)$ for every positive $n$. Then there exist elements $s(1), s(2), \cdots, s(n), s(n+1), \cdots$ in $S$ such that $p=\sum_{i=1}^{* n} a(i) \oplus t(n)$ for every positive $n$ where $t(n)$ is the sum of all the elements $s$ in $S$ which are different from $s(1), \cdots, s(n)$.

This may be deduced quite easily (by complete induction) from Lemma 3 . Note that Corollary 2 asserts among other things the infinity of the set $S$.

Lemma 4. Suppose that $p$ is refinable and that $p=\sum_{s \in S}^{*} s=\sum_{t \in T}^{*} t$ where the elements in $S$ and in $T$ are indecomposable.

(a) There exists to every finite subset $F$ of $S$ a subset $F^{*}$ of $T$ with the following properties:

(a.1) $F$ and $F^{*}$ contain the same number of elements.

(a.2) If $f$ is the sum of the elements in $F$ and $f^{*}$ is the sum of those elements in $T$ which do not belong to $F^{*}$, then $p=f \oplus f^{*}$.

(b) If $F, F^{*}, f$ and $f^{*}$ are determined as under (a), and if $t$ is an element in $T$ such that $o<f$, then $t$ belongs to $F^{*}$.

(c) $S$ and $T$ contain the same number of elements.

Proof. (a) is an immediate consequence of Lemma 3. If $F, F^{*}, f$ and $f^{*}$ are determined as under (a), and if $t$ does belong to $T$, but not to $F^{*}$, then $t \leqq f^{*}$ and hence $f t \leqq f f^{*}=o$, proving (b).

If $S$ is finite, then there exists, by (a), a subset $S^{*}$ of $T$ which contains as many elements as $S$ and which has the property $p=p \oplus q$ where $q$ is the (direct) sum of all the elements in $T$ which do not belong to $S^{*}$. Hence $q=o$, proving that $T=S^{*}$; and so $S$ and $T$ contain the same number of elements, if $S$ is finite.

Assume next that $S$ is infinite. Then $S$ contains an $n$-element subset for every positive integer $n$. It follows from (a) that $T$ contains an $n$-element subset for every $n$. Hence $T$ is infinite too. Denote by $c$ the cardinal number of the set $S$. Since $S$ is infinite, $c$ is likewise the cardinal number of the set of all finite subsets of $S$. Define to every finite subset $F$ of $S$ a finite subset $F^{*}$ of $T$ meeting requirements (a.1) and (a.2) which is possible by (a). This mapping $F \rightarrow F^{*}$ is a single-valued mapping of the set $F(S)$ of all the finite subsets of $S$ upon some subset $F(S)^{*}$ of the set $F(T)$ of all the finite subsets of $T$. It is clear that the cardinal number $c^{*}$ of $F(S)^{*}$ does not exceed the cardinal num- 
ber $c$ of $F(S)$. If $t$ is an element in $T$, then it follows from $o<t \leqq p=\sum_{s \in S}^{*} s$ and Postulate IV that there exists a finite subset $F_{t}$ of $S$ whose $\operatorname{sum} f_{t}$ satisfies $o<t f_{t}$. It follows from (b) that $t$ belongs to $\left(F_{t}\right)^{*}$. Thus we have shown that every element in $T$ belongs to at least one of the finite sets in $F(S)^{*}$. Since $T$ is infinite, and since the sets in $F(S)^{*}$ are finite (and cover $T$ ), it follows that the cardinal number $c^{*}$ of $F(S)^{*}$ is also the cardinal number of $T$. Thus we have shown that the cardinal number of $T$ does not exceed the cardinal number of $S$. Since our hypotheses are symmetric in $S$ and $T$, it follows likewise that the cardinal number of $S$ does not exceed the cardinal number of $T$. Thus $S$ and $T$ contain the same number of elements $\left({ }^{8}\right)$, completing the proof.

As an application we prove the following result.

THEOREM 1. If $p$ is refinable, if $p=\sum_{s \in S}^{*} s$ where the elements in $S$ are indecomposable, and if $p=a(1) \oplus \cdots \oplus a(i) \oplus \cdots$ where the elements $a(i)$ are indecomposable and form a finite or countably infinite set, then $S$ contains as many elements as there are elements $a(i)$; and the elements in $S$ may be numbered $s(1), \cdots, s(i), \cdots$ in such a way that

$$
p=\sum_{i=1}^{n} * a(i) \oplus \sum_{n<i}^{*} s(i) \quad \text { for every (admissible) } n .
$$

REMARK. A similar result has been established by Kiokemeister [1] for operator groups, meeting certain special requirements.

Proof. By Lemma 3 and Corollary 2 there exist elements $s(1), \cdots$, $s(i), \cdots$ in $S$ such that

$$
p=\sum_{i=1}^{n} a(i) \oplus t(n) \quad \text { for every (admissible) } n
$$

where $t(n)$ is the (direct) sum of the elements different from $s(1), \cdots, s(n)$ in $S$. If $s$ is an element in $S$, then $o<s \leqq p=\sum_{i}^{*} a(i)$ and we infer from Postulate IV the existence of an (admissible) positive integer $n$ such that $o<s \sum_{i=1}^{* n} a(i)$. Then it follows from Lemma $4(\mathrm{~b})$ that $s$ is one of the elements $s(1), \cdots, s(n)$; and thus we have shown that $s(1), \cdots, s(i), \cdots$ is an enumeration of the elements in $S$. That the number of elements $a(i)$ equals the number of elements in $S$ is a restatement of Lemma 4 (c).

C. Exchangeatility and equivalence.

Definition 2. The elements $u$ and $v$ are p-exchangeable if there exists an element $w$ such that $p=u \oplus w=w \oplus v$.

REMARK 1. If $u \oplus w=w \oplus v=p$, then it follows from $\$ 1$, Lemma 1 that $u+v=u \oplus w(u+v)=w(u+v) \oplus v$. Thus $p$-exchangeability of $u$ and $v$ implies $(u+v)$-exchangeability of $u$ and $v$. The following example shows that the converse is not true. Let $G$ be an ordinary finite abelian group, namely

$\left(^{8}\right)$ A similar argument is nowadays often used for proving Steinitz's theorem on the invariance of the degree of transcendency. 
$G=\{u\} \oplus\{v\}$ where $u$ is of order a prime $p$ and $v$ of order $p^{2}$. Then $\{u\}$ $\oplus\{u-p v\}=\{u-p v\} \oplus\{p v\}$, proving the "absolute" exchangeability of $\{u\}$ and $\{p v\}$. But it is readily seen that they are not $G$-exchangeable.

REMARK 2. If $u$ and $v$ are $q$-exchangeable, and if $q$ is a direct summand of $p$, then $u$ and $v$ are $p$-exchangeable. This is an immediate consequence of $\S 1$, Lemma 2.

REMARK 3. In $\$ 1$, Lemma 3 and the attached remark we have explained that $p$-exchangeability implies "center isomorphy."

The relation of $p$-exchangeability is symmetric and reflexive, but, in general, not transitive. Consequently we use the following definition.

Definition 3. The elements $u$ and $v$ are p-equivalent if there exists a finite number of elements $u=a(0), a(1), \cdots, a(n), a(n+1)=v$ such that $a(i)$ and $a(i+1)$ for $0 \leqq i \leqq n$ are $p$-exchangeable.

This implies the existence of elements $b(i)$ for $0 \leqq i \leqq n$ such that

$$
p=a(i) \oplus b(i)=b(i) \oplus a(i+1) \text { for every } i
$$

The relations of $p$-exchangeability and $p$-equivalence have been defined for direct summands of $p$ only. The equivalence relation is clearly symmetric, reflexive and transitive. Analogous relations have been defined in various circumstances (projective and continuous geometry and so on).

REMARK 4. It is a consequence of $\$ 1$, Corollary 1 that $a \oplus w=w \oplus b$ implies the isomorphy of $a$ and $b$. Consequently p-equivalent elements are isomorphic. Furthermore we may deduce from $\S 1$, Lemma 3 that $p$-equivalence of $u$ and $v$ implies $z(p)+u=z(p)+v$ (center isomorphy).

REMARK 5. If $P$ is the set of all the normal and admissible subloops of the operator loop $L$, then $L$-equivalence of $U$ and $V$ is known (Baer $[1$, p. 69, Corollary 2]) to imply (operator) center isomorphy of $U$ and $V$ (in a very strict sense).

The following proposition shows that in some very important special cases the situation regarding equivalence, and so on, is much simpler.

LEMMA 5. If $p$ is refinable, then the following properties of the indecomposable direct summands $u$ and $v$ of $p$ imply each other.

(i) $u$ and $v$ are $(u+v)$-exchangeable.

(ii) $u$ and $v$ are $p$-exchangeable.

(iii) $u$ and $v$ are p-equivalent.

Proof. In Remark 1 we have pointed out already that (ii) implies (i); and it is obvious that (ii) implies (iii).

Assume now that (i) is valid. Since $u$ and $v$ are direct summands of $p$, there exist elements $a$ and $b$ such that

$$
p=u \oplus a=v \oplus b \text {. }
$$

Since $p$ is refinable, there exist decompositions 


$$
u=u^{\prime} \oplus u^{\prime \prime}, \quad v=v^{\prime} \oplus v^{\prime \prime}, \quad a=a^{\prime} \oplus a^{\prime \prime}, \quad b=b^{\prime} \oplus b^{\prime \prime}
$$

such that

$$
\begin{aligned}
& p^{\prime}=u^{\prime} \oplus a^{\prime}=a^{\prime} \oplus v^{\prime}=v^{\prime} \oplus b^{\prime}=b^{\prime} \oplus u^{\prime}, \\
& p^{\prime \prime}=u^{\prime \prime} \oplus a^{\prime \prime}=a^{\prime \prime} \oplus b^{\prime \prime}=b^{\prime \prime} \oplus v^{\prime \prime}=v^{\prime \prime} \oplus u^{\prime \prime} ;
\end{aligned}
$$

and we have clearly ( $\$ 1$ Lemma 2$)$

$$
p=p^{\prime} \oplus p^{\prime \prime} .
$$

We distinguish two cases.

Case 1. $u^{\prime}=v^{\prime}=o$. Then it follows from $\left(3^{\prime \prime}\right)$ and (2) that

$$
p^{\prime \prime}=v \oplus u \text {. }
$$

But it follows from (3) that $p^{\prime \prime}$ is a direct summand of $p$, and (i) implies the $p^{\prime \prime}$-exchangeability of $u$ and $v$. Hence $u$ and $v$ are $p$-exchangeable.

Case 2. $u^{\prime}$ and $v^{\prime}$ are not both $o$. Then it follows from $\left(3^{\prime}\right)$ that neither $u^{\prime}$ nor $v^{\prime}$ is 0 , and it follows from the indecomposability of $u$ and $v$ that $u=u^{\prime}$, $v=v^{\prime}$. It follows from $\left(3^{\prime}\right)$ that

$$
p^{\prime}=u \oplus a^{\prime}=a^{\prime} \oplus v ;
$$

and thus $u$ and $v$ are $p^{\prime}$-exchangeable. But $p^{\prime}$ is a direct summand of $p$ by (3); and thus we have shown again that $u$ and $v$ are $p$-exchangeable. This completes the proof of the equivalence of (i) and (ii).

We precede the proof of the equivalence of (ii) and (iii) by the proof of the following simple proposition.

(4) If $q$ is refinable, if $q=h \oplus k=m \oplus n$ where $h, k, m, n$ are indecomposable, then either

$$
q=k \oplus m=h \oplus n
$$

or

$$
q=h \oplus m=k \oplus n
$$

or both are true.

From the refinability of $q$ we infer the existence of direct decompositions

$$
h=h^{\prime} \oplus h^{\prime \prime}, \quad k=k^{\prime} \oplus k^{\prime \prime}, \quad m=m^{\prime} \oplus m^{\prime \prime}, \quad n=n^{\prime} \oplus n^{\prime \prime}
$$

such that

$$
\begin{aligned}
q^{\prime} & =h^{\prime} \oplus k^{\prime}=k^{\prime} \oplus m^{\prime}=m^{\prime} \oplus n^{\prime}=n^{\prime} \oplus h^{\prime}, \\
q^{\prime \prime} & =h^{\prime \prime} \oplus k^{\prime \prime}=k^{\prime \prime} \oplus n^{\prime \prime}=n^{\prime \prime} \oplus m^{\prime \prime}=m^{\prime \prime} \oplus h^{\prime \prime} \\
q & =q^{\prime} \oplus q^{\prime \prime}
\end{aligned}
$$

We distinguish two cases. 
Case 1. $h^{\prime}=m^{\prime}=o$. Then $q^{\prime}=k^{\prime}=n^{\prime}$. If $q^{\prime}$ were not $o$, then it would follow from the indecomposability of $k$ and $n$ that $k^{\prime \prime}=n^{\prime \prime}=o$ and this would imply $m \oplus h=q^{\prime \prime}=o$, an impossibility. Hence $k^{\prime}=n^{\prime}=0$ and it follows from (4.2') that $\left(4^{\prime \prime}\right)$ is true.

Case 2. $h^{\prime}$ and $m^{\prime}$ are not both $o$. Then it follows from $\left(4.2^{\prime}\right)$ that none of them is $o$. Such $h$ and $m$ are indecomposable, since this implies $h=h^{\prime}, h^{\prime \prime}=0$ and $m=m^{\prime}, m^{\prime \prime}=0$. Now it follows from $\left(4.2^{\prime \prime}\right)$ that $n^{\prime \prime}=k^{\prime \prime}=0$ and hence $n=n^{\prime}, k=k^{\prime}$; and $\left(4^{\prime}\right)$ is a consequence of $\left(4.2^{\prime}\right)$. This completes the proof of (4).

We have to prove finally that (iii) implies (ii). Clearly it suffices to prove the following proposition which asserts transitivity of p-exchangeability.

(5) If $r, s, t$ are indecomposable elements such that

$$
p=r \oplus d=d \oplus s=s \oplus e=e \oplus t,
$$

then there exists an element $f$ such that

$$
p=r \oplus f=f \oplus t \text {. }
$$

We note first that we may choose $f=d$, if $p=d \oplus t$, and that we may choose $f=e$ in case $p=r \oplus e$. Thus we may assume now that

(5.3) $p$ is neither the direct sum of $d$ and $t$ nor the direct sum of $r$ and $e$.

Since $p=r \oplus d=e \oplus t$ is refinable, there exist direct decompositions

$$
r=r^{\prime} \oplus r^{\prime \prime}, \quad d=d^{\prime} \oplus d^{\prime \prime}, \quad e=e^{\prime} \oplus e^{\prime \prime}, \quad t=t^{\prime} \oplus t^{\prime \prime}
$$

such that

$$
\begin{aligned}
& p^{\prime}=r^{\prime} \oplus d^{\prime}=d^{\prime} \oplus e^{\prime}=e^{\prime} \oplus t^{\prime}=t^{\prime} \oplus r^{\prime}, \\
& p^{\prime \prime}=r^{\prime \prime} \oplus d^{\prime \prime}=d^{\prime \prime} \oplus t^{\prime \prime}=t^{\prime \prime} \oplus e^{\prime \prime}=e^{\prime \prime} \oplus r^{\prime \prime}
\end{aligned}
$$

Next we prove.

$$
p^{\prime}=r \oplus d^{\prime}=d^{\prime} \oplus e^{\prime}=e^{\prime} \oplus t=t \oplus r .
$$

If $r^{\prime}=o$, then it would follow from $\left(5.4^{\prime}\right)$ that $e^{\prime}=o$. If $p^{\prime}=t^{\prime} \neq o$, then $t=t^{\prime}, t^{\prime \prime}=o$ and this would imply $r=r^{\prime \prime}=o$ by $\left(5.4^{\prime \prime}\right)$, an impossibility. Hence $p^{\prime}=o$ and it would follow from (5.4') that $p=p^{\prime \prime}=e \oplus r$, contradicting (5.3). Consequently $r^{\prime} \neq 0$, and it follows from the indecomposability of $r$ that $r=r^{\prime}$ and $r^{\prime \prime}=o$. We infer from $\left(5.4^{\prime \prime}\right)$ that $t^{\prime \prime}=o$ and so $t=t^{\prime}$. Hence (5.5) is a consequence of $\left(5.4^{\prime}\right)$. We note that we have shown

$$
p^{\prime \prime}=d^{\prime \prime}=e^{\prime \prime} \text {. }
$$

We let $s^{*}=p^{\prime}\left(p^{\prime \prime}+s\right)$ and prove

$$
p^{\prime}=d^{\prime} \oplus s^{*}=s^{*} \oplus e^{\prime}
$$

From $p=d \oplus s=d^{\prime} \oplus\left(d^{\prime \prime} \oplus s\right)$ we infer that $d^{\prime} s^{*}=d^{\prime} p^{\prime}\left(p^{\prime \prime}+s\right)=d^{\prime}\left(d^{\prime \prime}+s\right)$ $=o$, since $p^{\prime \prime}=d^{\prime \prime}$ by $\left(5.5^{\prime}\right)$, and thus it follows from Dedekind's law that 


$$
\begin{aligned}
d^{\prime} \oplus s^{*} & =d^{\prime} \oplus p^{\prime}\left(p^{\prime \prime}+s\right)=p^{\prime}\left(d^{\prime}+d^{\prime \prime}+s\right)=p^{\prime}(d+s) \\
& =p^{\prime} p=p^{\prime}
\end{aligned}
$$

$p^{\prime}=s^{*} \oplus e^{\prime}$ is shown likewise.

We deduce now from $\left(5.5^{\prime}\right),(5.6)$ and $\$ 1$, Corollary 1 the isomorphy of $r, e^{\prime}, s^{*}, d^{\prime}$ and $t$ and so all these elements are indecomposable. We may consequently apply (4) on the direct decompositions

$$
p^{\prime}=r \oplus d^{\prime}=s^{*} \oplus e^{\prime}
$$

of the refinable element $p^{\prime}$ (which is refinable as a direct summand of the refinable element $p$ ). Consequently

$$
p^{\prime}=r \oplus s^{*}=d^{\prime} \oplus e^{\prime} \quad \text { or } \quad p^{\prime}=r \oplus e^{\prime}=d^{\prime} \oplus s^{*} .
$$

Since the second possibility would imply

$$
p=p^{\prime} \oplus p^{\prime \prime}=r \oplus e^{\prime} \oplus e^{\prime \prime}=r \oplus e
$$

by $\left(5.5^{\prime}\right)$, the second possibility leads to a contradiction with $(5.3)$; hence it follows that

$$
p=p^{\prime} \oplus p^{\prime \prime}=r \oplus\left(s^{*} \oplus p^{\prime \prime}\right) .
$$

Applying (4) likewise onto the decompositions

$$
p^{\prime}=t \oplus e^{\prime}=s^{*} \oplus d^{\prime}
$$

we find that

$$
p^{\prime}=t \oplus s^{*}=e^{\prime} \oplus d^{\prime} \quad \text { or } \quad p^{\prime}=t \oplus d^{\prime}=e^{\prime} \oplus s^{*} .
$$

The second possibility leads again to a contradiction with (5.3), since it would imply $p=p^{\prime} \oplus p^{\prime \prime}=t \oplus d^{\prime} \oplus d^{\prime \prime}=t \oplus d$. Consequently we have

$$
p=p^{\prime} \oplus p^{\prime \prime}=t \oplus\left(s^{*} \oplus p^{\prime \prime}\right) \text {. }
$$

From (5.7) and (5.8) we deduce the validity of (5), completing the proof.

LEMMA 6. If $u$ and $v$ are p-equivalent, then every direct summand of $u$ is p-equivalent to a direct summand of $v$.

Proof. It clearly suffices to prove the following proposition:

(6.1) If $u$ and $v$ are p-exchangeable, then every direct summand of $u$ is $p$ exchangeable against a direct summand of $v$.

From the hypothesis of (6.1) we infer the existence of an element $w$ such that $u \oplus w=w \oplus v=p$. Suppose now that $u=r \oplus s$. Let $x^{*}=v(w+x)$ for $x=r, s$. It follows from $\$ 1$, Corollary 2 that $v=r^{*} \oplus s^{*}$ and $w \oplus x=w \oplus x^{*}$ for $x=r, s$. Hence $p=r \oplus(s \oplus w)=(r \oplus w) \oplus s=\left(r^{*} \oplus w\right) \oplus s=r^{*} \oplus(w \oplus s)$ by $\$ 1$, Lemma 2, proving that $r$ and $r^{*}$ are $p$-exchangeable.

LEMMA 7. If $p=a \oplus b$ is refinable, then every indecomposable direct summand 
of $p$ is p-exchangeable against a direct summand of $a$ or of $b$.

Proof. Suppose that $p=d \oplus e$ and that $d$ is indecomposable. We infer from the refinability of $p$ the existence of decompositions

$$
a=a^{\prime} \oplus a^{\prime \prime}, \quad b=b^{\prime} \oplus b^{\prime \prime}, \quad d=d^{\prime} \oplus d^{\prime \prime}, \quad e=e^{\prime} \oplus e^{\prime \prime}
$$

such that

$$
\begin{aligned}
p^{\prime} & =a^{\prime} \oplus b^{\prime}=b^{\prime} \oplus d^{\prime}=d^{\prime} \oplus e^{\prime}=e^{\prime} \oplus a^{\prime}, \\
p^{\prime \prime} & =a^{\prime \prime} \oplus b^{\prime \prime}=b^{\prime \prime} \oplus e^{\prime \prime}=e^{\prime \prime} \oplus d^{\prime \prime}=d^{\prime \prime} \oplus a^{\prime \prime}, \\
p & =p^{\prime} \oplus p^{\prime \prime} .
\end{aligned}
$$

Since $d$ is indecomposable, either $d^{\prime}$ or $d^{\prime \prime}$ is $o$. If $d^{\prime}=o$, then $d=d^{\prime \prime}$ and it follows from (2) and $\left(2^{\prime \prime}\right)$ that

$$
\begin{aligned}
p=p^{\prime} \oplus p^{\prime \prime} & =\left(p^{\prime} \oplus e^{\prime \prime}\right) \oplus d \\
& =\left(p^{\prime} \oplus e^{\prime \prime}\right) \oplus b^{\prime \prime}
\end{aligned}
$$

and $d$ is $p$-exchangeable against the direct summand $b^{\prime \prime}$ of $b$. If $d^{\prime \prime}=o$ then $d=d^{\prime}$ and it follows from (2), (2') that

$$
\begin{aligned}
p=p^{\prime \prime} \oplus p^{\prime} & =\left(p^{\prime \prime} \oplus b^{\prime}\right) \oplus d \\
& =\left(p^{\prime \prime} \oplus b^{\prime}\right) \oplus a^{\prime}
\end{aligned}
$$

and $d$ is $p$-exchangeable against the direct summand $a^{\prime}$ of $a$.

D. Exchange of blocks.

DeFinition 4. The direct summands $u$ and $v$ of $p$ are relatively prime direct summands of $p$, if p-equivalence of the direct summands $h$ and $k$ of $u$ and $v$ respectively implies $h=k=0$.

LEMMA 8. If the refinable element $p$ is the direct sum of indecomposable elements, and if $u$ and $v$ are direct summands of $p$ such that no indecomposable direct summand of $u$ is p-exchangeable against a direct summand of $v$, then $u$ and $v$ are relatively prime direct summands of $p$.

Proof. Suppose that the direct summands $h$ and $k$ of $u$ and $v$ respectively are $p$-equivalent. If $h$ were not $o$, then it would follow from Lemma 2 that there exists an indecomposable direct summand $d$ of the direct summand $h$ of $p$. Since $h$ and $k$ are $p$-equivalent, we infer from Lemma 6 the existence of a direct summand $e$ of $k$ such that $d$ and $e$ are $p$-equivalent. Since $d$ and $e$ are $p$-equivalent direct summands of $p$, the indecomposability of $d$ implies the indecomposability of $e$, and it follows from Lemma 5 that $d$ and $e$ are $p$ exchangeable. But $d$ is a direct summand of $u$ and $e$ is a direct summand of $v$; and thus the hypothesis $h \neq o$ has led us to a contradiction. Hence $h=o$. Since $h$ and $k$ are equivalent, $k=o$, proving that $u$ and $v$ are relatively prime.

LEMMA 9. If the refinable element $p=a \oplus b=d \oplus e$, and if $a$ and $e$ are relatively 
prime direct summands of $p$, then the following properties imply each other.

(i) $p=a \oplus e$.

(ii) $p=b \oplus d$.

(iii) $d$ and $b$ are relatively prime direct summands of $p$.

Proof. Assume first the validity of (iii). Because of the refinability of $p=a \oplus b=d \oplus e$ there exist direct decompositions

$$
a=a^{\prime} \oplus a^{\prime \prime}, \quad b=b^{\prime} \oplus b^{\prime \prime}, \quad \cdot d=d^{\prime} \oplus d^{\prime \prime}, \quad e=e^{\prime} \oplus e^{\prime \prime}
$$

such that

$$
\begin{aligned}
p^{\prime} & =a^{\prime} \oplus b^{\prime}=b^{\prime} \oplus d^{\prime}=d^{\prime} \oplus e^{\prime}=e^{\prime} \oplus a^{\prime}, \\
p^{\prime \prime} & =a^{\prime \prime} \oplus b^{\prime \prime}=b^{\prime \prime} \oplus e^{\prime \prime}=e^{\prime \prime} \oplus d^{\prime \prime}=d^{\prime \prime} \oplus a^{\prime \prime}, \\
p & =p^{\prime} \oplus p^{\prime \prime} .
\end{aligned}
$$

From (1) and (1") we infer that $a^{\prime \prime}$ and $e^{\prime \prime}$ are $p$-exchangeable direct summands of $a$ and $e$ respectively; and that $b^{\prime \prime}$ and $d^{\prime \prime}$ are $p$-exchangeable direct summands of $b$ and $d$ respectively. Since $a$ and $e$ are relatively prime direct summands of $p$, we have $a^{\prime \prime}=e^{\prime \prime}=o$; and since $b$ and $d$ are relatively prime direct summands of $p$, we have $b^{\prime \prime}=d^{\prime \prime}=o$. Hence $p^{\prime \prime}=o$ too; and it fullows from (1), (1') that

$$
p=a \oplus b=b \oplus d=d \oplus e=e \oplus a .
$$

Thus we have shown that both (i) and (ii) are consequences of (iii).

Assume next the validity of (i); and suppose that $h$ and $k$ are $p$-equivalent direct summands of $b$ and $d$ respectively. We have $p=a \oplus b=a \oplus e$ and $p=e \oplus d=e \oplus a$. Hence $b$ and $e$ are $p$-equivalent, and it follows from Lemma 6 that $h$ is $p$-equivalent to a direct summand $h^{*}$ of $e$. Likewise $d$ and $a$ are $p$ equivalent and $k$ is $p$-equivalent to a direct summand $k^{*}$ of $a$. Thus $h^{*}$ and $k^{*}$ are $p$-equivalent direct summands of $e$ and $a$. Since $e$ and $a$ are relatively prime it follows that $h^{*}=k^{*}=0$. Since $p$-equivalent elements are isomorphic, this implies $h=k=0$, proving that (iii) is a consequence of (i).

Assume finally the validity of (ii). Then we have $p=b \oplus a=b \oplus d$ and $p=d \oplus e=d \oplus b$. Thus $a$ and $d$ are $p$-exchangeable direct summands of $p$; and $b$ and $e$ are $p$-exchangeable direct summands of $b$. Arguing as in the preceding paragraph we see that $b$ and $d$ are relatively prime, completing the proof.

LEMMA 10. If the refinable element $p$ is the direct sum of indecomposable elements, if $p=a \oplus b=d \oplus e$, and if $a, e$ and $d, b$ are pairs of relatively prime direct summands of $p$, then $a, b$ and $d$, $e$ are pairs of relatively prime direct summands of $p$.

Proof. Suppose there exists a pair $h, k$ of $p$-exchangeable indecomposable direct summands of $a$ and $b$ respectively. It follows from Lemma 7 that $h$ is $p$-exchangeable against a direct summand of $d$ or of $e$. Since $a$ and $e$ are rela- 
tively prime, and since $h \neq o$, it is impossible that $h$ is $p$-exchangeable against a direct summand of $e$. Hence $h$ is $p$-exchangeable against a direct summand $m$ of $d$. It follows that $k$ and $m$ are $p$-equivalent direct summands of $b$ and $d$ respectively. But $k \neq 0$ and $b$ and $d$ are relatively prime. Thus we have been led to a contradiction, proving that there does not exist a pair of $p$-exchangeable indecomposable direct summands of $a$ and $b$. It follows from Lemma 8 that $a$ and $b$ are relatively prime direct summands of $p$; and it follows likewise that $d$ and $e$ are relatively prime.

Notation. If $p$ is the direct sum of the elements in $S$, and if $d$ is a direct summand of $p$, then $(S, d)$ is the direct sum of all those elements in $S$ which are $p$ exchangeable against $d$ and $(S, d)^{\prime}$ is the direct sum of all those elements in $S$ which are not p-exchangeable against $d$.

It is clear that $p=(S, d) \oplus(S, d)^{\prime}$. We note that one of these components may be $o$. We shall use these decompositions only in case $d$, as well as the elements in $S$, is indecomposable.

Lemma 11. Suppose that $p$ is refinable, $p=\sum_{s \in S}^{*} s=\sum_{t \in T^{t}}^{*}$ and that $d$, the elements $s$ in $S$ and $t$ in $T$, are indecomposable direct summands of $p$. Then $(S, d)$ and $(T, d)$ are not $o$ and $(S, d)$ and $(T, d)^{\prime}$ are relatively prime direct summand of $p$.

Proof. It is a consequence of Lemma 1 that $d$ may be exchanged against an element in $S$ (in $T$ ), proving that neither $(S, d)$ nor $(T, d)$ is $o$. Suppose now that $h$ and $k$ are $p$-exchangeable indecomposable direct summands of $(S, d)$ and $(T, d)^{\prime}$ respectively. Then it follows from Lemma 1 that $h$ is $p$-exchangeable against an element $s$ in $S$ which is $p$-exchangeable against $d$, and that $k$ is $p$-exchangeable against an element $t$ in $T$ which is not $p$-exchangeable against $d$. Consequently $d$ and $t$ are $p$-equivalent, and it follows from Lemma 5 that they are $p$-exchangeable, a contradiction. Thus there does not exist a pair of $p$-exchangeable indecomposable direct summands of $(S, d)$ and $(T, d)^{\prime}$ respectively, and hence it follows from Lemma 8 that $(S, d)$ and $(T, d)^{\prime}$ are relatively prime direct summands of $p$.

We are now ready to prove the principal results of $\$ 2$.

ThEOREM 2. Suppose that the refinable element $p=\sum_{s \in S}^{*} s=\sum_{t \in T}^{*} t$, that the elements in $S$ and in $T$ are indecomposable and that $d$ is some indecomposable direct summand of $p$. Then

(a) $p=(S, d) \oplus(S, d)^{\prime}=(S, d) \oplus(T, d)^{\prime}=(T, d) \oplus(S, d)^{\prime}=(T, d) \oplus(T, d)^{\prime}$;

(b) $S$ and $T$ contain the same number of elements p-exchangeable against $d$.

Proof. $p=(S, d) \oplus(S, d)^{\prime}=(T, d) \oplus(T, d)^{\prime}$, as has been pointed out before. It follows from Lemma 11 that $(S, d),(T, d)^{\prime}$ and $(T, d),(S, d)^{\prime}$ are pairs of relatively prime direct summands of $p$. Hence we infer (a) from Lemma 9.

Denote by $X(d)$ the set of all the elements in $X$ which are $p$-equivalent to $d$. Then $(S, d)$ is the direct sum of $S(d)$ and $(T, d)$ the direct sum of $T(d)$. 
Let $(S, d)^{\prime}=w$. Then, by (a), $p=w \oplus(S, d)=w \oplus(T, d)$. Let $x^{*}=(S, d)(w+x)$ for $x$ in $T(d)$. Then it follows from $\$ 1$, Corollary 2 that $w \oplus x=w \oplus x^{*}$ and that $(S, d)$ is the direct sum of the $x^{*}$ for $x$ in $T(d)$. From the indecomposability of $x$ we infer the indecomposability of $x^{*}$. Since $(S, d)$ is refinable (as a direct summand of $p$ ) and is a direct sum of indecomposable elements, it follows from Lemma 4 , (c) that $S(d)$ contains as many elements as there are elements $x^{*}$ for $x$ in $T(d)$. Hence $S(d)$ and $T(d)$ contain the same number of elements, proving (b).

DefinItIon 5. An isomorphism of the direct decomposition $S$ of $p$ upon the direct decomposition $T$ of $p$ is a one-to-one mapping of $S$ upon $T$ such that corresponding elements are p-exchangeable.

This is not the weakest definition of isomorphism which is possible, since we could substitute for " $p$-exchangeable" the word " $p$-equivalent" which would, in general, lead to a weaker definition of isomorphy. In the present context it does not make any difference because of Lemma 5. Note that Theorem 1 asserts a fairly strong form of isomorphy, stronger than the present one.

Theorem 3. Any two direct decompositions of a refinable element into indecomposable direct summands are isomorphic.

This is an immediate consequence of Theorem 2.

REMARK 1. Though Theorem 3 is more concise, it should not be overlooked that Theorem 2 has more content.

REMARK 2. If the operator loop $L$ is refinable, and if $L=\sum_{\mu}{ }^{*} S(\mu)=\sum_{\nu}^{*} T(\nu)$ are two direct decompositions of $L$ into indecomposable direct summands, then we infer from Theorem 3 the possibility of numbering the $T$ 's in such a way that $S(\mu)$ and $T(\mu)$ are exchangeable. But exchangeability implies center isomorphy (Baer $[1$, p. 69]); now it is clear how to construct a center automorphism of $L$ which maps $S(\mu)$ upon $T(\mu)$. We may restate this result:

Any two direct decompositions of the refinable operator loop $L$ into indecomposable direct summands are center isomorphic.

3. Refinement theorems. In the present section we shall combine $\$ 1$, Lemma 5 with $\S 1$, Theorem 3 and $\S 2$, Theorem 3 to obtain generalizations of the latter theorems.

A. Existence of center free blocks.

LEMMA 1. If $p=\sum_{s \in S} s$, then there exists a greatest subset $M$ of $S$ such that $o=z(p) \sum_{s \in M} S$.

Proof. There exist certainly subsets $N$ of $S$ such that $o=z(p) \sum_{s \in N} s$; for instance the vacuous set. To prove our contention it suffices therefore to verify the following statement: $o=z(p) \sum_{s \in T} s$, if $o=z(p)[t(1)+\cdots+t(n)]$ for every finite subset $t(1), \cdots, t(n)$ of $T$; and this last proposition is an immediate consequence of Postulate IV. 
Lемма 2. If $p$ is the direct sum of the subset $S$ of $P$, and if the ascending or descending chain condition is satisfied by the partially ordered set of elements $x \leqq z(p)$, then there exists a finite subset $s(1), \cdots, s(n)$ of $S$ such that $o=z(p) t$ where $t$ is the sum of elements different from $s(1), \cdots, s(n)$ in $S$.

Proof. If $F$ is a finite subset of $S$, then we denote by $s(F)$ the sum of the elements in $F$ and by $s(F)^{\prime}$ the sum of the elements in $S$, but not in $F$. It follows from $\$ 1$, Lemma 2 that $p=s(F) \oplus s(F)^{\prime}$.

Assume first the validity of the ascending chain condition in $z(p)$. Then there exists in the set of elements $z(p) s(F)$, for $F$ a finite subset of $S$, a greatest element, say $z(p) s(W)$. If $z(p) s(W)^{\prime}$ were not $o$, then we would infer from Postulate IV and $o<z(p) s(W)^{\prime} \leqq s(W)^{\prime}$ the existence of a finite number of elements $s(1), \cdots, s(k)$ in $S$ none of which belongs to $W$ such that $o<z(p) \sum_{i=1}^{* k} s(i)$. Denote by $V$ the set composed of the elements $s(i)$ and the elements in $W$. Then $s(W) \leqq s(V)$. Since $V$ is a finite subset of $S$, it follows from the maximality of $z(p) s(W)$ that $z(p) s(W)=z(p) s(V)$. This implies in particular that $z(p) \sum_{i=1}^{* k} s(i) \leqq z(p) s(W)$. Consequently we have, by $\S 1$, Lemma 2 ,

$$
o<z(p) \sum_{i=1}^{k} * s(i)=z(p) s(W) \sum_{i=1}^{k} * s(i)=o,
$$

a contradiction which proves that $z(p) s(W)^{\prime}=0$.

Assume next the validity of the descending chain condition in $z(p)$. Then there exists in the set of elements $z(p) s(F)^{\prime}$, for $F$ a finite subset of $S$, a smallest element, say $z(p) s(M)^{\prime}$. If $z(p) s(M)^{\prime}$ were not $o$, then there would exist, by IV, elements $t(1), \cdots, t(m)$ in $S$ none of of which is in $M$ such that $o<z(p) \sum_{i=1}^{* m} t(i)$. If $N$ is the finite subset of $S$ which is composed of the $t(i)$ and the elements in $M$, then $s(N)^{\prime} \leqq s(M)^{\prime}$; and it follows from the minimality of $z(p) s(M)^{\prime}$ that $z(p) s(M)^{\prime}=z(p) s(N)^{\prime}$. It follows from $\S 1$, Lemma 2 that $s(M)^{\prime}=s(N)^{\prime} \oplus \sum_{i=1}^{* m} t(i)$. Hence

$$
o<z(p) \sum_{i=1}^{m} t(i)=z(p) s(N)^{\prime} \sum_{i=1}^{m} * t(i)=o,
$$

a contradiction which proves that $z(p) s(M)^{\prime}=o$. This completes the proof.

B. Existence of isomorphic refinements. It has been pointed out that isomorphy of direct decompositions may be defined in a variety of ways. In this section we shall need, apart from the isomorphy according to $\$ 2$, Definition 5, a somewhat stronger form.

DEFINITION 1. If $p=\sum_{s \in S}^{*} s=\sum_{t \in T}^{*}$, and if $\alpha$ is a one-to-one mapping of $S$ upon $T$ such that $p=s^{\alpha} \oplus s^{\prime}$ for every $s$ in $S$, then $\alpha$ is an exchange isomorphism of the decomposition $S$ upon the decomposition $T$.

As usual $s^{\prime}$ indicates the sum of all the elements, not $s$, in $S$.

It is clear that $s$ and $s^{\alpha}$ are $p$-exchangeable; and so exchange isomorphy 
implies isomorphy in the sense of $\$ 2$, Definition 5 . Note that $\S 2$, Theorem 1 contains another form of isomorphy between direct decompositions. In general, neither inverses nor products of exchange isomorphies need be exchange isomorphies $\left({ }^{9}\right)$.

DEFINITION 2. If $p$ is the direct sum of $S$, and if every element $s$ in $S$ is the direct sum of the set $T(s)$, then the direct decomposition of $p$ into the join $T$ of the sets $T(s)$ is a refinement of $S$.

That $p$ is the direct sum of $T$ is a consequence of $\$ 1$, Lemma 2 .

For instance, $\S 1$, Theorem 3 assures the existence of exchange isomorphic refinements of two given direct decompositions into a finite number of direct summands. We are going to prove the following two propositions.

THEOREM 1. If the element $p$ is refinable, and if the ascending or the descending chain condition is satisfied by $z(p)$, then any two direct decompositions of $p$ possess exchange isomorphic refinements.

Theorem 2. If $p=\sum_{s \in S}^{*} s=\sum_{l \in T}^{*}$ is refinable, if $o=f(S) z(p)=f(T) z(p)$ where $f(X)$ is, for $X=S, T$, the sum of all those elements in $X$ which are not indecomposable, then the direct decompositions $S$ and $T$ of $p$ possess isomorphic refinements.

These theorems may be considered as generalizations of results due to Golowin [1] and Kurosh [1, 2].

It should be noted that when applied on operator loops either of these theorems assures the existence of center isomorphic refinements to given direct decompositions.

Finally it may be worth noting that Theorem 2 contains $\$ 2$, Theorem 3 as a special case.

The main step of the proof of either theorem is the derivation of a certain lemma which is valid without any hypotheses concerning the element $p$. Accordingly we precede the proofs proper of these propositions by two general discussions which may be of interest independent of their ultimate objective.

A. We shall deduce a number of properties from the following hypotheses.

$$
p=a \oplus b=d \oplus e \quad \text { and } \quad b z(p)=e z(p)=o .
$$

It follows from $\S 1$, Lemma 5 that

$$
a=a d \oplus a e, \quad d=d a \oplus d b .
$$

Consequently $p=a d \oplus a e \oplus b=d a \oplus d b \oplus e$; and hence it follows from Dedekind's law (or $\$ 1$, Lemma 1) that

$$
b=d b \oplus b(d a \oplus e), \quad e=a e \oplus e(a d \oplus b) .
$$

Next we deduce from Dedekind's law that

${ }^{(9)}$ See in this context Baer $[1$, p. 85, Remark 3]. 

$a d \oplus b(d a \oplus e)=(a d \oplus b)(a d \oplus e)=a d \oplus e(a d \oplus b)$.

Substituting (A.1) and (A.2) into our decompositions of $p$ we find that

$$
\begin{aligned}
p & =a d \oplus a e \oplus b=a d \oplus a e \oplus d b \oplus b(d a \oplus e) \\
& =d a \oplus d b \oplus e=d a \oplus d b \oplus a e \oplus e(a d \oplus b) .
\end{aligned}
$$

It follows readily from (A.1) and (A.4) that

$$
a+d=a d \oplus a e \oplus d b,
$$

and we infer from (A.3) and (A.4) that

$$
p=d b \oplus a e \oplus(a d \oplus b)(a d \oplus e) .
$$

B. Next we prove the following fundamental proposition.

LEMMA. If $p=a \oplus b=d \oplus e$, if $o=b z(p)=e z(p)$, and if

$$
a=\sum_{h \in H}^{*} h, \quad b=\sum_{k \in K}^{*} k, \quad d=\sum_{m \in M}^{*} m, \quad e=\sum_{n \in N}^{*} n,
$$

then

(1) $\quad a d=\sum_{m \in M}^{*} a m=\sum_{h \in H} * d h$;

$h=d h \oplus \sum_{n \in N}^{*} h\left(n+\sum_{x} d x\right)$ for $h$ in $H$ where $x$ ranges over the elements, not $h$, in $H$,

$k=\sum_{m \in M}^{*} k(m+a d) \oplus \sum_{n \in N}^{*} k(n+a d)$ for $k$ in $K$,

$m=a m \oplus \sum_{k \in K}^{*} m\left(k+\sum_{\nu} a y\right)$ for $m$ in $M$ where y ranges over the

elements, not $m$, in $M$,

$n=\sum_{h \in I I} * n(h+a d) \oplus \sum_{k \in K}^{*} n(k+a d) \quad$ for $n$ in $N$;

$a d \oplus(k+a d) n=a d \oplus(n+a d) k$ for $k$ in $K$ and $n$ in $N$, $a d \oplus\left(k+\sum_{y} a y\right) m=a d \oplus(m+a d) k$ for $k$ in $K, m$ in $M$ where $y$

(3) ranges over the elements, not $m$, in $M$, $a d \oplus(h+a d) n=a d \oplus\left(n+\sum_{x} d x\right) h$ for $h$ in $H, n$ in $N$ where $x$ ranges over the elements, not $h$, in $H$. 
Proof of the lemma. From $o=b z(p), p=\sum_{m \in M}^{*} m \oplus \sum_{n \in N}^{*} n$ and $\$ 1$, Lemma 5 we infer that $a=\sum_{m \in M}^{*} a m \oplus \sum_{n \in N}^{*} a n$. Since $m \leqq d, o=n d$, we infer from Dedekind's law that

$$
a d=\sum_{m \in M}^{*} a m+\sum_{n \in N}^{*} a n, \quad d=\sum_{m \in M} *_{M} a m ;
$$

from $n \leqq e, m e=o$ we infer similarly that $a e=\sum_{n \in N}^{*} a n$. If we interchange at the same time (as we may) $a$ and $d, b$ and $e$ and so on, we verify the validity of the following formulas:

$$
a d=\sum_{m \in M}^{*} a m=\sum_{h \in H} * d h, \quad a e=\sum_{n \in N}^{*} a n, \quad d b=\sum_{k \in K}^{*} d k .
$$

Thus we have shown in particular the validity of (1).

It follows from (A.4) that $p=(a d \oplus b) \oplus a e=\sum_{m \in M}^{*} m \oplus \sum_{n \in N}^{*} n$. Since $a e z(p)=0$, we may now apply $\$ 1$, Lemma 5 ; hence it follows that $a d \oplus b$ $=\sum_{m \in M}^{*}(a d+b) m \oplus \sum_{n \in N}^{*}(a d+b) n$. From $m \leqq d$ and Dedekind's law it follows that

$$
(a d+b) m=(a d+b) d m=(a d+b d) m=d m=m \text { by (A.1). }
$$

Consequently we have

$$
a d \oplus b=\sum_{m \in M}^{*} m \oplus \sum_{n \in N}^{*}(a d+b) n=d \oplus \sum_{n \in N} *(a d+b) n .
$$

It follows from (A.1) to (A.3) that

$a d \oplus b=a d \oplus d b \oplus b(d a \oplus e)=d b \oplus a d \oplus e(d a+b)=d \oplus e(d a+b)$.

Since $(a d+b) n \leqq(a d+b) e$, we inf er from

$$
d \oplus e(d a+b)=a d \oplus b=d \oplus \sum_{n \in N}^{*}(a d+b) n
$$

that

$$
e(d a+b)=\sum_{n \in N}^{*}(a d+b) n, \quad b(a d+e)=\sum_{k \in K}^{*}(a d+e) k
$$

where, as before, the second equation may be deduced from the first one by reasons of symmetry.

We infer from (5) and Dedekind's law that

$$
\begin{aligned}
a d \oplus \sum_{n \in N}^{*}(a d+b) n & =a d \oplus e(a d+b)=(a d+e)(a d+b) \\
& =a d \oplus b(a d+e) ;
\end{aligned}
$$

and likewise we see that

$$
a d \oplus(a d+b) n=(a d+n)(a d+b)=a d \oplus(a d+n) b
$$

so that 


$$
a d \oplus b(a d+e)=a d \oplus \sum_{n \in N}^{*}(a d+b) n=a d \oplus \sum_{n \in N}^{*}(a d+n) b .
$$

Now we infer from $(a d+n) b \leqq(a d+e) b$ that

$$
b(a d+e)=\sum_{n \in N}^{*}(a d+n) b, \quad e(d a+b)=\sum_{k \in K}^{*}(a d+k) e
$$

where the second equation may be deduced from the first one by the symmetry argument.

It follows from $\$ 1$, Lemma 4 that $z[b(a d+e)] \leqq(a d+e) b z(p)=o$, since $b(a d+e)$ is, by (A.2), a direct summand of $p$. It follows from $\S 1$, Theorem 1 that $b(a d+e)$ is $S$-refinable; and hence it follows from $\$ 1$, Theorem 2 and (5), $\left(5^{\prime}\right)$ that

$$
b(a d+e)=\sum_{k, n}^{*}(a d+e) k(a d+n) b .
$$

From $k \leqq b$ and $a d+n \leqq a d+e$ we infer now that

$$
b(a d+e)=\sum_{k, n}^{*} k(a d+n), \quad e(d a+b)=\sum_{k, n}^{*} n(d a+k)
$$

where the double sums range over every $k$ in $K$ and every $n$ in $N$ and where the second equation is deduced from the first one by symmetry arguments.

From $k=k b, n=n e$ and (6) one deduces immediately that

$$
k(a d+e)=\sum_{n \in N}^{*} k(a d+n), \quad n(d a+b)=\sum_{k \in K}^{*} n(d a+k)
$$

for every $k$ in $K$ and $n$ in $N$.

Next we prove:

$$
a e=\sum_{h \in H}^{*} e(h+a d), \quad d b=\sum_{m \in M}^{*} b(m+a d) .
$$

For reasons of symmetry it suffices to prove the first of these equations. We denote by $h^{\prime}$, for $h$ in $H$, the sum of all the $x \neq h$ in $H$; and we denote by $h^{\prime \prime}$, for $h$ in $H$, the sum of all the $d x$ for $x \neq h$ in $H$. We note that $a=h \oplus h^{\prime}$ and that $h^{\prime \prime} \leqq d h^{\prime}$. First we find that

$$
\begin{aligned}
a d \oplus e(h+a d) \sum_{x} e(x+a d) & =(h+a d)\left[a d+\sum_{x} e(x+a d)\right] \\
& =(h+a d) \sum_{x}(a d+e)(x+a d) \\
& =(h+a d)\left[a d+\sum_{x} x(e+a d)\right]
\end{aligned}
$$

where $h$ is in $H$ and where $x$ ranges over all the elements in $H$ with the exception of $h$. Consequently 


$$
x(e+a d)=x a(e+a d)=x(a e+a d)=x
$$

by $x \leqq a$, Dedekind's law and (A.1). The above expression is therefore equal to

$$
\begin{array}{rlrl}
(h+a d)\left(a d+h^{\prime}\right) & =a d+h^{\prime}(h+a d) & & \text { (by Dedekind's law) } \\
& =a d+h^{\prime}\left(h+d h+h^{\prime \prime}\right) & \text { (by (4)) } \\
& =a d+h^{\prime} h+h^{\prime \prime} & & \text { (by Dedekind's law, since } \left.h^{\prime \prime} \leqq h^{\prime}\right) \\
& =a d & & \text { (since } h h^{\prime}=o \text { and } h^{\prime \prime} \leqq a d \text { by (4). }
\end{array}
$$

This implies that $o=e(h+a d) \sum_{x} e(x+a d)$, implying in particular that the sum of the $e(h+a d)$ is their direct sum. Next we verify that

$$
\begin{array}{rlr}
a d \oplus \sum_{h \in H}^{*} e(h+a d) & =\sum_{h \in H}(a d+e)(a d+h) & \text { (by Dedekind's law) } \\
& =\sum_{h \in H}(a d+e) a(a d+h) & \text { (since } h \leqq a) \\
& =\sum_{h \in H}(a d+a e)(a d+h) \\
& =\sum_{h \in H}(a d+h) & \text { (by Dedekind's law) } \\
& =a d+\sum_{h \in H} h=a d+a=a=a d \oplus a e & \text { (by (A.1)) }
\end{array}
$$

this implies

$$
a e=\sum_{h \in H}^{*} e(h+a d),
$$

completing the proof of (8).

$$
\begin{array}{ll}
k=\sum_{m}^{*} k(m+a d) \oplus \sum_{n \in N}^{*} k(n+a d) & \text { for } k \text { in } K, \\
n=\sum_{h \in H}^{*} n(h+a d) \oplus \sum_{k \in K}^{*} n(k+a d) & \text { for } n \text { in } N .
\end{array}
$$

For reasons of symmetry it suffices to prove the second of these equalities. We infer from (A.2), (8) and (5') that

$$
\sum_{n \in N}^{*} n=e=a e \oplus e(a d \oplus b)=\sum_{h \in H}^{*} e(h+a d) \oplus \sum_{k \in K}^{*} e(k+a d) .
$$

Since $e$ is a direct summand of $p$, it follows from $\$ 1$, Lemma 4 that $z(e)$ $\leqq e z(p)=o$. It follows from $\S 1$, Theorems 1 and 2 that

$$
\begin{aligned}
e & =\sum_{h, n}^{*} n e(h+a d) \oplus \sum_{k, n}^{*} n e(k+a d) \\
& =\sum_{h, n}^{*} n(h+a d) \oplus \sum_{k, n}^{*} n(k+a d) \\
& =\sum_{n \in N} \sum_{h, k}^{*}[n(h+a d) \oplus n(k+a d)],
\end{aligned}
$$


where the summation ranges over all the $h$ in $H, k$ in $K$ and $n$ in $N$. From $n e=n$ one deduces now readily our contention (9).

$$
a d \oplus n(k+a d)=a d \oplus k(n+a d) \quad \text { for } n \text { in } N \text { and } k \text { in } K .
$$

This is immediately deduced from Dedekind's law, since both sides of this equation are equal to $(a d+n)(a d+k)$, and since furthermore $d n=a k=0$.

$$
a e=\sum_{h, n} * n(h+a d), \quad d b=\sum_{m, k}^{*} k(m+a d)
$$

where the summations range over all the $h$ in $H, k$ in $K, m$ in $M, n$ in $N$.

It follows from (9) and Dedekind's law that

$$
a n=\sum_{h \in H} * n(h+a d) \oplus a \sum_{k \in K}^{*} n(k+a d),
$$

since $h+a d \leqq a$. It follows from (7) that

$$
a \sum_{k \in K}^{*} n(k+a d)=a n(d a+b)=n(d a+a b)=n d a=o
$$

by Dedekind's law and $o=a b=n d$. Thus we have shown that

$$
a n=\sum_{h \in H}^{*} n(h+a d) \text {. }
$$

But it follows from (4) that $a e=\sum_{n \in N}^{*} a n$; thus the first equation (11) is a consequence from $\left(11^{\prime}\right)$, and the second equation follows by reasons of symmetry.

(12) $h=d h \oplus \sum_{n \in N}^{*} h\left(n+\sum_{x} d x\right)$ for $h$ in $H$ where $x$ ranges over all the elements in $H$, not $h$; and

$m=a m \oplus \sum_{k \in K}^{*} m\left(k+\sum_{\nu} a y\right)$ for $m$ in $M$ where $y$ ranges over all the elements in $M$, not $m$.

It suffices again to prove the first of these equations. We have

$$
\begin{aligned}
\sum_{h \in H} *\left[d h+\sum_{n \in N} h\left(n+\sum_{x} d x\right)\right] \\
=\sum_{h \in H} * d h+\sum_{h, n} h\left(n+\sum_{x} d x\right) \\
=a d+\sum_{h, n} h\left(n+\sum_{x} d x\right) \\
=\sum_{h, n}\left[a d+d h+h\left(n+\sum_{x} d x\right)\right] \\
=\sum_{h, n}\left[a d+h\left(d h+n+\sum_{x} d x\right)\right]
\end{aligned}
$$

(by Dedekind's law) 


$$
\begin{aligned}
& =\sum_{h, n}[a d+h(a d+n)] \\
& =\sum_{h, n}(a d+h)(a d+n)=\sum_{h, n}[a d+n(a d+h)] \\
& =a d+\sum_{h, n} n(a d+h)=a d+a e \\
& =a \\
& =\sum_{h \in H}^{*} h,
\end{aligned}
$$

and from this equation we infer $h=d h+\sum_{n \in N} h\left(n+\sum_{x} d x\right)$.

Letting, as always, $x$ range over all the elements in $H$ with the exception of $h$, we deduce from Dedekind's law that

$$
\begin{aligned}
d h \sum_{h \in N} h\left(n+\sum_{x} d x\right) & \leqq h d\left(e+\sum_{x} d x\right) \\
& =h\left(d e+\sum_{x} d x\right)=h \sum_{x} d x=0 .
\end{aligned}
$$

If $n$ is some fixed element in $N$, and if $y$ ranges over all the elements in $N$ with the exception of $n$, then we have

$$
\begin{aligned}
h\left(n+\sum_{x} d x\right)\left[d h+\sum_{y} h\left(y+\sum_{x} d x\right)\right] & \quad \text { (by Dedekind's law) } \\
= & h\left(n+\sum_{x} d x\right) \sum_{y}\left(d h+y+\sum_{x} d x\right) \quad \text { (by (4)) } \\
= & h\left(n+\sum_{x} d x\right) \sum_{y}(y+d a) \\
& =h\left(n+\sum_{x} d x\right)\left(d a+\sum_{y} y\right) \\
& =h\left[\sum_{x} d x+n\left(d a+\sum_{y} y\right)\right] \\
& =h \sum_{x} d x=o,
\end{aligned}
$$

since $n \leqq e$ and $e d=o, n y=0$, and so on. Thus we have shown that the above sum is a direct sum, completing the proof of (12).

This completes the proof of (2). The first equation (3) we have already verified; see (10). Of the other two equations (3) it suffices to verify one for reasons of symmetry. If $k$ is in $K, m$ in $M$, and if $y$ ranges over the elements, not $m$, in $M$, then 


$$
\begin{aligned}
a d m\left(k+\sum_{y} a y\right) & =a m\left(k+\sum_{y} a y\right) & & \\
& =m\left(a k+\sum_{y} a y\right) & & \text { (by Dedekind's law) } \\
& =m \sum_{y} a y=o, & & \\
a d(m+a d) k & =o, & & \text { since } a k \leqq a b=o ;
\end{aligned}
$$

hence

$$
\begin{aligned}
a d \oplus\left(k+\sum_{y} a y\right) m & =a m \oplus \sum_{y} a y \oplus\left(k+\sum_{y} a y\right) m \quad \text { (by (4)) } \\
& =\sum_{y} a y+\left(a m+k+\sum_{y} a y\right) m \text { (by Dedekind's law) } \\
& =\sum_{y} a y+(k+a d) m=(k+a d)\left(m+\sum_{y} a y\right) \\
& =(k+a d)\left(m+a m+\sum_{y} a y\right) \\
& =(k+a d)(m+a d)=a d \oplus k(m+a d),
\end{aligned}
$$

completing the proof of (3) and of the lemma.

REMARK. If we add to the hypotheses $o=b z(p)=e z(p)$ the further hypothesis $o=a d$, then Lemma (2) assures the existence of common refinements of the given direct decompositions.

C. Proof of Theorem 1. Assume that $p=\sum_{s \in S}^{*} s=\sum_{t \in T}^{*} t$ is refinable and that the ascending or the descending chain condition is satisfied by $z(p)$. We infer from Lemma 2 the existence of finite subsets $H$ and $M$ of $S$ and $T$ respectively with the following properties: If $K$ is the set of elements in $S$, but not in $H$, and $b$ the sum of $K$, then $o=b z(p)$; if $N$ is the set of elements in $T$, but not in $M$, and $e$ the sum of $N$, then $o=e z(p)$.

Denote by $a$ and $d$ the sums of $H$ and $M$ respectively. Then it follows from (A.1) that $a d$ is a direct summand of $p$. Since $p$ is refinable, so is $a d$. It follows from Lemma B, (1) that

$$
a d=\sum_{h \in H}^{*} d h=\sum_{m \in M}^{*} a m
$$

are two direct decompositions of the refinable element $a d$ into a finite number of direct summands each. It follows from $\S 1$, Theorem 3 that these two direct decompositions of ad possess isomorphic refinements. Now the existence of isomorphic refinements to the two given direct decompositions $S$ and $T$ of $p$ is an immediate consequence of Lemma B. 
D. Proof of Theorem 2. Assume that $p=\sum_{s \in S}^{*} S=\sum_{t \in T}^{*} t$ is refinable. Denote by $H$ the set of the indecomposable elements in $S$, by $K$ the set of the other elements in $S$; and by $M$ the set of the indecomposable elements in $T$ and by $N$ the set of the other elements in $T$. Denote by $a, b, d, e$ the sum of $H, K, M, N$ respectively. Assume that $o=b z(p)=e z(p)$. It follows from (A.1) that $a d$ is a direct summand of $p$. Since $p$ is refinable, so is $a d$. It follows from Lemma B, (1) that

$$
a d=\sum_{h \in H}^{*} d h=\sum_{m \in M}^{*} a m .
$$

It follows from Lemma B, (2) that $d h$ is a direct summand of the indecomposable element $h$. Hence we have either $d h=o$ or $d h=d$; and likewise we see that either $a m=o$ or $a m=m$. Thus, omitting in the decompositions (D) the terms that are equal to $o$ (as we may), we have obtained in (D) two direct decompositions of the refinable element ad into indecomposable elements. It follows from $\$ 2$, Theorem 3 that these two direct decompositions (D) of $a d$ are isomorphic. Now the existence of isomorphic refinements to the two given direct decompositions $S$ and $T$ of $p$ is an immediate consequence of Lemma B.

REMARK. Note that the refinability of $p$ is only used to prove refinability of the direct summand $a d$. Note furthermore that by using $\$ 2$, Theorem 2 instead of $\S 2$, Theorem 3 a slight improvement of Theorem 2 would have been possible.

R. BAER

\section{BIBLIOGRAPHY}

1. Direct decompositions, Trans. Amer. Math. Soc. vol. 61 (1947) pp. 62-98.

G. BIRKHOFF

1. Lattice theory, Amer. Math. Soc. Colloquium Publications, vol. 25, New York, 1940.

O. N. GoLowis

1. On factors without centers in direct decompositions of groups, Rec. Math. (Mat. Sbornik) N.S. vol. 6 (1939) pp. 423-426.

B. JónsSON AND A. TARSKI

1. Direct decompositions of finite algebraic systems, Notre Dame Mathematical Lectures, No. 5, 1947.

F. KIOKEMEISTER

1. A note on the Remak-Schmidt theorem, Bull. Amer. Math. Soc. vol. 53 (1947) pp. 957958.

A. KUROSH

1. Isomor phisms of direct decompositions I, Bull. Acad. Sci. URSS. Ser. Math. vol. 7 (1943) pp. 185-202.

2. Isomorphisms of direct decompositions II, Bull. Acad. Sci. URSS. Sér. Math. vol. 10 O. ORE (1946) pp. 47-72.

1. Direct decompositions, Duke Math. J. vol. 2 (1936) pp. 581-596.

UNIVERSITY OF ILLINOIS, Urbana, IlL. 